\title{
Leptogenesis from a sneutrino condensate revisited
}

\author{
Rouzbeh Allahverdi ${ }^{a}$ and Manuel Drees ${ }^{b}$ \\ ${ }^{a}$ Theory Group, TRIUMF, 4004 Wesbrook Mall, Vancouver, B.C., V6T 2A3, Canada. \\ ${ }^{b}$ Physik Department, Technische Universität München, James Franck Strasse, D85748, Garching, Germany.
}

(November 8, 2018)

\begin{abstract}
We re-examine leptogenesis from a right-handed sneutrino condensate, paying special attention to the $B$-term associated with the see-saw Majorana mass. This term generates a lepton asymmetry in the condensate whose time average vanishes. However, a net asymmetry will result if the sneutrino lifetime is not much longer than the period of oscillations. Supersymmetry breaking by thermal effects then yields a lepton asymmetry in the standard model sector after the condensate decays. We explore different possibilities by taking account of both the low-energy and Hubble $B$-terms. It will be shown that the desired baryon asymmetry of the Universe can be obtained for a wide range of Majorana mass.
\end{abstract}

\section{INTRODUCTION}

The baryon asymmetry of the Universe (BAU) parameterized as $\eta_{\mathrm{B}}=\left(n_{\mathrm{B}}-n_{\overline{\mathrm{B}}}\right) / s$, with $s$ being the entropy density, is determined to be $0.9 \times 10^{-10}$, with a precision of $\sim 4 \%$, by the recent WMAP data on the temperature anisotropy in cosmic microwave background (CMB) [1]. This is also in good agreement with an independent determination from big bang nucleosynthesis (BBN) [2] of the light elements. This asymmetry can be produced from a baryon symmetric Universe provided three conditions are met: $B$ and/or $L$-violation, $C-$ and $C P$-violation, and departure from thermal equilibrium [3]. Moreover, $B+L$-violating sphaleron transitions are active at temperatures $T$ from $10^{12} \mathrm{GeV}$ down to 100 $\mathrm{GeV}$ [4]. Any mechanism for creating a baryon asymmetry at $T>100 \mathrm{GeV}$ therefore has to create a $B-L$ asymmetry. The final baryon asymmetry is then given by $B=a(B-L)$, where $a=28 / 79$ in case of the standard model (SM) and $a=32 / 92$ for the minimal supersymmetric standard model (MSSM) [5].

Leptogenesis is an attractive mechanism for producing a $B-L$ asymmetry [6]. This scheme postulates the existence of right-handed $(\mathrm{RH})$ neutrinos, which are SM singlets, with a lepton number violating Majorana mass $M_{N}$. Such a mass is compatible with all SM symmetries, and hence can be arbitrarily large beyond the electroweak scale. This provides an elegant explanation for the small masses of the light neutrinos via the see-saw mechanism [7]. Moreover, a lepton asymmetry can be generated from the out-of-equilibrium decay of the $\mathrm{RH}$ neutrinos to the Higgs boson and light leptons, provided $C P$-violating phases exist in the neutrino Yukawa couplings. The lepton asymmetry thus obtained will be partially converted to a baryon asymmetry via sphaleron effects. This is the standard lore for leptogenesis $[6,8,9]$. In this scenario the on-shell RH neutrinos whose decay is responsible for the lepton asymmetry can be produced thermally or non-thermally. In thermal leptogenesis the $\mathrm{RH}$ neutrinos are produced from the primordial thermal bath through their Yukawa interactions. However, at least one RH neutrino must have small Yukawa couplings in order to decay sufficiently late, i.e. out of thermal equilibrium [9]. The generation of an acceptable lepton asymmetry then requires the mass $M_{1}$ of the lightest RH neutrino and the temperature of the thermal bath to exceed $10^{8} \mathrm{GeV}$ [10-12] (unless RH neutrinos are degenerate [13]).

However, this is marginally compatible with the upper bound on the reheat temperature $T_{\mathrm{R}}$ in supersymmetric theories, which is constrained by thermal gravitino production [14]. Gravitinos with mass $m_{3 / 2}$ of the order of the electroweak scale decay long after BBN and their decay products can distort the primordial abundance of the light elements. For $100 \mathrm{GeV} \lesssim m_{3 / 2} \lesssim 1 \mathrm{TeV}$, a successful nucleosynthesis requires $n_{3 / 2} / s \leq\left(10^{-14}-10^{-12}\right)$, which translates into the bound $T_{\mathrm{R}} \leq\left(10^{7}-10^{9}\right) \mathrm{GeV}$ on the reheat temperature $[14,15]^{*}$.

An interesting alternative is non-thermal leptogenesis. In this scenario RH neutrinos are produced from the decay of the inflaton [21], and the reheat temperature can be significantly below $M_{N}$. This can occur for a perturbative inflaton decay, called reheating, provided that the RH neutrinos are lighter than the inflaton [22]. The RH neutrinos can also be produced non-perturbatively via preheating [23], or tachyonic preheating [24], even if their mass is larger than the inflaton mass. Non-thermal leptogenesis can also be achieved without exciting onshell RH neutrinos [25-27]. This allows a sufficiently low reheat temperature, and can yield the required baryon asymmetry for a rather wide range of the inflationary scale.

\footnotetext{
*Non-thermal gravitino production during preheating [16] does not give rise to any threat in realistic models of inflation $[17,18]$. Also, possible gravitino production from perturbative decays of the inflaton [19], and/or from heavy longlived neutral particles [20] will not yield severe bounds.
} 
In supersymmetric models one also has the $\mathrm{RH}$ sneutrinos which serve as an additional source for leptogenesis [28]. The sneutrinos are produced along with neutrinos in a thermal bath or during reheating, and with much higher abundances in preheating [29]. Moreover, there are two unique possibilities for leptogenesis from the RH sneutrinos. First, they can acquire a large vacuum expectation value (VEV) if their mass during inflation is less than the Hubble expansion rate at that epoch $H_{I}$. This condensate starts oscillating once $H \simeq M_{N}$, thereby automatically satisfying the out-of-equilibrium condition. The decay of the sneutrino condensate can then yield the desired lepton asymmetry in the same fashion as neutrino decay does [30,31], or [32] via the Affleck-Dine mechanism [33,34]. The second possibility is to generate the lepton asymmetry in the $\mathrm{RH}$ sneutrino sector $[35-37]^{\dagger}$. This can be done via inflaton decay to the RH sneutrinos [35], or from soft supersymmetry breaking effects [36,37]. This asymmetry will be transferred to the light (s)leptons upon the decay of the $\mathrm{RH}$ sneutrinos, and partially converted to baryon asymmetry via sphalerons. However, as emphasized in $[36,37]$, the final asymmetry depends on the strength of supersymmetry breaking effects and will vanish in the supersymmetric limit.

In this note we re-examine the generation of a lepton asymmetry from a RH sneutrino condensate in the light of new proposals. We will focus on the role of the soft supersymmetry breaking $B$-term associated with the neutrino Majorana mass. This term creates an oscillating asymmetry in the RH sneutrino condensate whose average, when taken over many oscillations, vanishes. However, the condensate does carry a lepton asymmetry at any given time, which can be accessed if the sneutrino lifetime is not much longer than the oscillation period. We will consider different cases by taking account of both the low-energy and Hubble $B$-terms. It will be shown that condensate decay can result in an acceptable BAU through supersymmetry breaking by statistics and finite temperature mass corrections. It is important for the success of this scenario that the sneutrino condensate is not destroyed before an $\mathcal{O}(1)$ asymmetry is produced by the $B$-term(s). We will consider two possible effects in this respect, namely resonant decay of the condensate and thermal effects. We will show that thermal effects can prevent resonant decay, while being sufficiently weak in order not to affect the condensate dynamics themselves. We will also comment on the possibility that the $\mathrm{RH}$ sneutrino is the inflaton or the curvaton, in which case the sneutrino condensate dominates the energy density at the time of its decay. Our main conclusion is that an acceptable baryon asymmetry can be obtained

\footnotetext{
${ }^{\dagger}$ Note that, due to the Majorana nature of the RH neutrinos, no lepton asymmetry can be created in that sector.
}

for wide ranges of the Majorana mass and $B$ term, either Hubble-induced or from low energy supersymmetry breaking. This therefore provides a viable alternative for successful leptogenesis from a sneutrino condensate, which works for a single generation and does not require any parameter in the Lagrangian to have a nontrivial phase.

The remainder of this article is organized as follows. In the next Section we discuss and solve the evolution equation of the sneutrino condensate in a matter-dominated universe. Sec. III deals with perturbative sneutrino decays, with emphasis on supersymmetry breaking by thermal effects. In Sec. IV we discuss possible nonperturbative decays of the $\tilde{N}$ condensate, and ways to shut them off; we also derive an upper bound on the reheat temperature from the requirement that scattering off the thermal bath does not destroy the coherence of the condensate. Numerical results leading to successful leptogenesis consistent with all constraints are presented in Sec. V, while Sec. VI contains a discussion of special features of our mechanism, and briefly sketches the consequences of loosening some of our assumptions. Finally, Sec. VII is devoted to a short summary and some conclusions.

\section{LEPTON ASYMMETRY IN THE SNEUTRINO CONDENSATE}

We work in the framework of the MSSM augmented with three RH neutrino multiplets in order to accommodate neutrino masses via the see-saw mechanism [7]. The relevant part of the superpotential is

$$
W \supset \frac{1}{2} M_{N} \mathbf{N N}+h \mathbf{H}_{u} \mathbf{N L},
$$

where $\mathbf{N}, \mathbf{H}_{u}$, and $\mathbf{L}$ are multiplets containing the $\mathrm{RH}$ neutrinos $N$ and sneutrinos $\tilde{N}$, the Higgs field giving mass to the top quark and its superpartner, and the left-handed (s)lepton doublets, respectively. $h$ are the neutrino Yukawa couplings and, for simplicity, family indices on $M_{N}, h, \mathbf{N}$, and $\mathbf{L}$ are omitted. We work in the basis where the Majorana mass matrix is diagonal. Note that we are not concerned with the origin of this mass. It can come from an explicit mass term or from spontaneous breaking of some symmetry, e.g. in models with a gauged $U(1)_{B-L}$.

In addition to the supersymmetry conserving part of the scalar potential for $\tilde{N}$, one also has soft terms from low-energy supersymmetry breaking [38]

$$
m_{0}^{2}|\tilde{N}|^{2}+\left(B M_{N} \tilde{N}^{2}+\text { h.c. }\right)
$$

and supersymmetry breaking by the energy density of the Universe (called Hubble-induced) [39]

$$
C_{I} H^{2}|\tilde{N}|^{2}+\left(b H M_{N} \tilde{N}^{2}+\text { h.c. }\right) \text {. }
$$


The soft supersymmetry breaking parameters $m_{0}$ and $B$ typically are $100 \mathrm{GeV}-1 \mathrm{TeV}$ at the weak scale. In models with gauge-mediated supersymmetry breaking, $B$ can be very small at the "messenger" scale where supersymmetry breaking is transmitted to the visible sector. For $\left|C_{I}\right| \sim \mathcal{O}(1)$ and $|b| \sim \mathcal{O}(1)$, Hubble-induced supersymmetry breaking is dominant as long as $H>m_{0}$; note that $H \gg m_{0}$ during inflation. It is known that all scalar fields with mass less than $H_{I}$ can acquire a VEV during inflation, due to the accumulation of quantum fluctuations [21]. Here we consider the case where $M_{N}<H_{I}{ }^{*}$. Therefore the flatness of the $\tilde{N}$ potential crucially depends on the sign and size of $C_{I}$. If $C_{I} \gtrsim 1, \tilde{N}$ will settle at the origin and a condensate will not be formed. For $0<C_{I} \ll 1$, quantum fluctuations will grow along the $\tilde{N}$ direction during inflation. These fluctuations can push $|\langle\tilde{N}\rangle|$ to a maximum value of $H_{I}^{2} / M_{N}[21]$. Higher VEVs are also possible as initial condition. On the other hand, if $C_{I}<0$, the origin is an unstable point and higher dimensional terms in the scalar potential set the minimum of $\tilde{N}$.

The only renormalizable superpotential term of this type is $\lambda \mathbf{N N N}$. However, this term violates $\mathrm{R}$-parity and, in consequence, destabilizes the lightest supersymmetric particle (LSP). The LSP is only a viable dark matter candidate if this term is much too small to be relevant in our discussion [40]. Another possibility at the renormalizable level will arise if $\mathbf{N}$ is charged under some new symmetry at a scale $M>M_{N}$ (e.g., in $S O(10)$ GUTs). In this case a $D$-term contribution $\sim g^{2}|\tilde{N}|^{4}$ appears at scales above $M$, with $g$ being a gauge coupling, leading to $|\langle\tilde{N}\rangle| \lesssim M$. It is also possible to have nonrenormalizable superpotential terms. When $\mathbf{N}$ remains a singlet up to very high scales terms like $\lambda_{n} \mathbf{N}^{n} / M^{n-3}$, with $n>3$ and $\lambda_{n} \sim \mathcal{O}(1)$, are allowed where $M$ is $M_{\mathrm{GUT}}$ or $M_{\mathrm{P}}^{\dagger}$. However, such terms can be dangerous (if $\lambda_{n}$ is not suppressed) as the potential develops other minima at very large $|\langle\tilde{N}\rangle|$. The sneutrino field may then get trapped in (one of) these minima if it initially has a larger VEV, thus violating $\mathrm{R}$-parity and destabilizing the lightest supersymmetric particle (LSP). If $\mathbf{N}$ is charged under some gauge group, these terms can arise only after spontaneous breaking of the new symmetry typically resulting in $\lambda_{n} \ll 1$. As an example, consider the $S O(10)$ GUT. Then $\lambda_{n}$ has powers of $M_{\mathrm{GUT}} / M_{\mathrm{P}}$ and/or $M_{N} / M_{\mathrm{GUT}}$. It is therefore expected that in general the $D$-term contribution limits $|\langle\tilde{N}\rangle|$ from above, if $\tilde{N}$ is a non-singlet.

In what follows we consider $\langle\tilde{N}\rangle$ at the end of inflation to be a free parameter. For $H>M_{N}$, the absolute

\footnotetext{
*Note that the RH (s)neutrinos can be massless during inflation. This happens, e.g., in the model of Ref. [32], where the Majorana mass term is generated via the Higgs mechanism after inflation.

${ }^{\dagger} M_{\mathrm{P}}=2.4 \times 10^{18} \mathrm{GeV}$ is the reduced Planck mass.
}

value of the $\mathrm{RH}$ sneutrino $|\tilde{N}|$ rolls down very slowly. It will start oscillating around the origin, with an initial amplitude $N_{0}$, when $H \simeq M_{N}$. The low energy and Hubble $B$-terms, from Eqs. (2) and (3), generate a potential for the phase of $\tilde{N}^{\ddagger}$. In general the phase will change if $\tilde{N}_{0}$ is displaced from the minima of this potential; this corresponds to an angular motion in the complex plane $(\Re \mathrm{e} \tilde{N}, \Im \mathrm{m} \tilde{N})$. This is generally the case since the phase dependence of $V$ is very weak for $H>M_{N}$, and hence $\arg \tilde{N}_{0}$ will generically be far away from the minima. The phase motion results in the creation of a lepton asymmetry in the $\tilde{N}$ sector, though with a big difference from the usual Affleck-Dine mechanism [33]. There lepton/baryon number violation is induced by new physics at a scale so high that its effects will appear only as nonrenormalizable interactions once heavy degrees of freedom are integrated out [41]. On the other hand, here the $\mathrm{RH}$ sneutrinos take part in the dynamics as their mass is below the inflationary scale. Therefore the lepton number violating effects, encoded in the $B$-terms, appear as renormalizable interactions. This has very important consequences for leptogenesis from an $\tilde{N}$ condensate.

A non-renormalizable $A$-term quickly becomes irrelevant: it multiplies a higher power of the relevant scalar field than the mass term does, and is thus redshifted more rapidly than the mass term by the expansion of the Universe. Therefore its only role is to trigger the phase motion of the condensate by providing an initial "torque". Once the $A$-term effectively disappears, due to the redshift, the scalar field VEV will freely "rotate" (i.e. its real and imaginary parts will oscillate with the same frequency) resulting in a constant lepton/baryon asymmetry [33]. In our case, on the other hand, the $B$-term is bilinear in $\tilde{N}$, and hence is redshifted in exactly the same way as the mass term. This implies that the $B$-term is always relevant and the motion along the angular direction will be oscillatory rather than free rotation (i.e. the real and imaginary parts will oscillate with different frequencies). In consequence, the lepton asymmetry created in the $\tilde{N}$ condensate is also oscillating coherently and its time average vanishes. A sizable net asymmetry can be obtained only if the condensate decay time is comparable to the period of oscillation of the lepton number carried by $\tilde{N}$, which is determined by the size of the $B$-terms.

We now turn to a quantitative description of the behavior of $\tilde{N} \equiv\left(\tilde{N}_{R}+i \tilde{N}_{I}\right) / \sqrt{2}$, where $\tilde{N}_{R, I}$ are real scalar fields. As already mentioned, a crucial role is played by the $B$-term, which we can take to be real (any phase

\footnotetext{
${ }^{\ddagger}$ It is important to notice that for $H \leq M_{N}$, nonrenormalizable superpotential terms and the corresponding low energy and Hubble $A$-terms are subdominant to the terms in Eqs. (1), (2) and (3).
} 
in $B$ can be absorbed* by a re-definition of $\tilde{N})$. It lifts the mass degeneracy between $\tilde{N}_{R}$ and $\tilde{N}_{I}$; allowing both Hubble-induced and low-energy SUSY breaking, these masses are given by

$$
\begin{aligned}
m_{R, I}^{2} & =M_{N}^{2}+C_{I} H^{2}+m_{0}^{2} \pm 2 M_{N}(B+b H) \\
& \simeq\left[M_{N} \pm(B+b H)\right]^{2},
\end{aligned}
$$

where the upper $(+)$ sign applies to $m_{R}^{2}$, and the second approximate equality holds for $M_{N} \gg B+b H, \sqrt{\left|C_{I}\right|} H$. The initial conditions at $H \simeq M_{N}$ can be chosen as

$\tilde{N}_{R}=N_{0} \cos \theta_{0} ; \tilde{N}_{I}=N_{0} \sin \theta_{0} ; \frac{d}{d t}\left(t \tilde{N}_{R}\right)=\frac{d}{d t}\left(t \tilde{N}_{I}\right)=0$,

where $\theta_{0}$ is a phase, which will generically be $\mathcal{O}(1)$. The fields then evolve following the standard equations of motion [42]:

$$
\ddot{\tilde{N}}_{R, I}+\left(3 H+\Gamma_{N}\right) \dot{\tilde{N}}_{R . I}+m_{R, I}^{2} \tilde{N}_{R, I}=0,
$$

where $m_{R, I}^{2}$ are as in Eq.(4), $\Gamma_{N}$ is the $\tilde{N}$ decay width, which we will compute in the next Section, and the dots denote differentiation with respect to time. Note that $\tilde{N}_{R}$ and $\tilde{N}_{I}$ evolve independently of each other.

As mentioned earlier, we assume that the Hubble parameter during inflation $H_{I} \gg M_{N}$. This means that $H \lesssim M_{N}$, the situation of interest to us, occurs first during the matter-dominated era, when the inflaton field oscillates coherently. Let us assume for the moment that $\tilde{N}$ decays before the inflaton does. Moreover, we assume that the total energy in the Universe is dominated by the inflaton; in Sec. VI F we will comment on scenarios where the sneutrino itself dominates the energy density. We then only need to solve Eq.(6) during the matterdominated era, where we can set $3 H=2 / t, t$ being the time. The late-time behavior of the solution of this equation can be given analytically:

$$
\begin{aligned}
\tilde{N}_{R, I}(t)=\frac{\mathrm{e}^{-\Gamma_{N}\left(t-t_{M}\right) / 2}}{t} & {\left[A_{R, I} \cos \left(f_{R, I}(t)\right)\right.} \\
& \left.+B_{R, I} \sin \left(f_{R, I}(t)\right)\right] .
\end{aligned}
$$

The functions $f_{R, I}$ are given by

$$
f_{R, I}(t)=\omega_{R, I}\left(t-t_{M}\right) \pm \frac{2 b M_{N}}{3 \omega_{R, I}} \ln \frac{t}{t_{M}}
$$

\footnotetext{
*A relative phase between $B$ and $b$ would modify the evolution of the $\tilde{N}$ condensate somewhat during the (rather short) period of time where $|B| \sim|b| H$. However, this will lead to qualitatively different behavior only in the highly unlikely case that $\tilde{N}_{0}$ is purely real or purely imaginary. We will therefore not pursue this possibility any further.
}

with

$$
\omega_{R, I}=\sqrt{M_{N}^{2}+m_{0}^{2} \pm 2 B M_{N}-\Gamma_{N}^{2} / 4} \simeq M_{N} \pm B .
$$

Eqs.(7)-(9) solve Eq.(6) up to terms of relative order $C_{I} /\left(M_{N} t\right)^{2}, b /\left(M_{N} t\right)^{2}, \quad \Gamma_{N} /\left(M_{N}^{2} t\right) .^{\dagger}$ The coefficients $A_{R, I}$ and $B_{R, I}$ can be obtained by matching Eq.(7) at $t=t_{M}$ to a full (numerical) solution of Eq.(6). If Hubbleinduced SUSY breaking is negligible, i.e. if $\left|C_{I}\right|,|b| \ll 1$, Eq.(7) can be used at all times $t \gtrsim 1 / M_{N}$; we will see in Sec. $\mathrm{V}$ that $\Gamma_{N}<10^{-6} M_{N}$ is required, i.e. the solution (7) of Eq.(6) becomes nearly exact in this case.

The lepton asymmetry in the $\tilde{N}$ sector is generally given by

$$
\begin{aligned}
L_{\tilde{N}} & \equiv n_{\tilde{N}}-n_{\tilde{N}^{*}}=i\left(\dot{\tilde{N}} * \tilde{N}-\dot{\tilde{N}} \tilde{N}^{*}\right) \\
& =\dot{\tilde{N}}_{I} \tilde{N}_{R}-\dot{\tilde{N}}_{R} \tilde{N}_{I} .
\end{aligned}
$$

Whenever $\tilde{N}$ is described by Eq.(7), the asymmetry in the $\tilde{N}$ condensate amounts to

$$
\begin{aligned}
& L_{\tilde{N}} \simeq \frac{\mathrm{e}^{-\Gamma_{N}\left(t-t_{M}\right)}}{t^{2}}\left\{\omega \left[\left(A_{R} B_{I}-B_{R} A_{I}\right) \cos \left(f_{R}-f_{I}\right)\right.\right. \\
& \left.+\left(A_{R} A_{I}+B_{R} B_{I}\right) \sin \left(f_{R}-f_{I}\right)\right] \\
& -\Delta\left[\left(A_{R} B_{I}+A_{I} B_{R}\right) \cos \left(f_{R}+f_{I}\right)\right. \\
& \left.\left.+\left(B_{R} B_{I}-A_{R} A_{I}\right) \sin \left(f_{R}+f_{I}\right)\right]\right\},
\end{aligned}
$$

with

$$
\Delta(t)=\delta+\frac{2 b M_{N}}{3 \omega t}
$$

Here we have used the notation $\omega_{R, I}=\omega \pm \delta$. As mentioned earlier, if Hubble-induced SUSY breaking is negligible, we can match the solution (7) directly to the initial condition (5). Ignoring the small, $\mathcal{O}(B)$ terms in the last two lines of Eq.(11), the lepton asymmetry of the condensate then becomes

$$
L_{\tilde{N}} \simeq \frac{\mathrm{e}^{-\Gamma_{N} t}}{t^{2}} M_{N} N_{0}^{2} \sin 2 \theta_{0} \sin (2 B t) .
$$

The factor $1 / t^{2}$ in Eqs.(11), (13) just describes the redshifting of the $\tilde{N}$ number density; it will drop out when we normalize the asymmetry to the inflaton or entropy density. Apart from this factor, the asymmetry in Eq.(13) reaches its maximum at $t \simeq(|B|)^{-1}$ but, due to its oscillation with a frequency $2|B|$, its average vanishes

${ }^{\dagger}$ The $1 / t$ factor in Eq.(7) explains why we chose vanishing time derivative for the "co-moving" fields $t \tilde{N}_{R, I}$ as initial condition (5): only in this case can $N_{0}$ be interpreted as initial amplitude of the oscillation of the $\tilde{N}$ field. More general boundary conditions give very similar results so long as the energy stored in the $\tilde{N}_{R, I}$ fields remains the same. 
over longer times. The RH sneutrino must therefore have a decay rate $\Gamma_{N} \simeq|B|$ in order to transfer the maximum lepton asymmetry to left-handed (s)leptons. This is a crucial point to which we will come back in the next section. The difference from the usual lepton/baryon asymmetry generation from a condensate is evident. A dimension three or higher $A$-term generates an asymmetry and becomes irrelevant soon thereafter, thus the (co-moving) asymmetry approaches a constant. In our case the asymmetry is generated by a dimension two $B$-term and has an oscillatory nature. The maximum asymmetry is obtained after a quarter of oscillation and can be transferred efficiently to the light (s)leptons only if $\tilde{N}$ condensate decays around the same time.

Eq.(11) shows that the behavior of the lepton asymmetry stored in the $\tilde{N}$ condensate becomes more complicated in the presence of a Hubble-induced $B$-term, since then the difference $f_{R}-f_{L}$ contains a term $\propto b \ln \frac{t}{t_{M}}$. If this term dominates, it leads to a period of oscillation that grows exponentially with time. If $b$ is $\mathcal{O}(1)$, this effectively sweeps a wide range of oscillation frequencies, and therefore allows efficient transfer of the asymmetry to light (s)lepton fields for any $\Gamma_{N} \in\left[|B|, M_{N} e^{-1 /|b|}\right]$. The size of $|b|$ depends on the details of the inflationary model and the inflaton coupling to the RH (s)neutrinos. Since $H \sim m_{\phi} \hat{\phi} / M_{\mathrm{P}}$, with $m_{\phi}$ being the inflaton mass and $\hat{\phi}$ the amplitude of inflaton oscillations, $|b|$ is linear in the inflaton field during the the matter-dominated era of inflaton oscillations. It should therefore vanish if forbidden by some symmetry (e.g., an R-symmetry), so long as that symmetry is unbroken. If the inflaton couples to the $\mathrm{RH}$ (s)neutrinos only gravitationally, one generally has $|b| \propto \phi_{0} / M_{\mathrm{P}}$, where $\phi_{0}$ is the inflaton VEV at the minimum of its potential [20]. Thus it is expected that $|b| \ll 1$ in models of chaotic inflation where $\phi_{0}=0$, and new inflation where $\phi_{0} \ll M_{\mathrm{P}}$ is possible. In such cases the Hubble $B$-term is probably negligible, otherwise it should be taken into account.

One comment is in order before moving on to the next section. Here we have assumed that $H_{u}$ and $\tilde{L}$ have a $\mathrm{VEV} \ll N_{0}$ at the end of inflation. Otherwise the situation will be considerably more complicated due to the coupling of these fields to the sneutrino, leading to the scenario studied in [43]. A natural way to achieve this is to make $H_{u}$ and $\tilde{L}$ sufficiently heavy by requiring $h N_{0}>H_{I}$. For an acceptable choice of $h$ and $N_{0}$ allowing successful baryogenesis (see next sections), a constraint on the scale of inflation will thus be obtained. It can be evaded if, for example, $H_{u}$ and $\tilde{L}$ have a Hubble-induced mass $^{2}>H^{2}$. This requires the soft mass of $\tilde{N}$ to be considerably different from that of $H_{u}$ and $\tilde{L}$ at scales of order $H_{I}{ }^{\ddagger}$

\footnotetext{
${ }^{\ddagger}$ Even if these masses are comparable at a renormalization scale near $M_{\mathrm{P}}$, they could differ significantly at scale $H_{I}$ [44].
}

\section{SNEUTRINO DECAY AND SUPERSYMMETRY BREAKING}

It is crucial to transfer the asymmetry generated in the $\tilde{N}$ condensate to left-handed (LH) (s)leptons, so that it will be partially converted into a baryon asymmetry by sphalerons. In general one can imagine two possibilities for creating a lepton/baryon asymmetry from a condensate. First, the asymmetry is produced in a condensate carrying lepton/baryon number and then transferred to fermions via lepton/baryon number conserving interactions. This is what happens in the Affleck-Dine mechanism [33]. The second possibility is that the condensate does not carry any lepton/baryon number but some other $U(1)$ charge. In this case the free rotation of the condensate amounts to an asymmetry in the $U(1)$ charge. This can be converted into lepton/baryon asymmetry provided the condensate decay to fermions violates lepton/baryon number. This possibility has been considered for baryogenesis from the decay of a complex inflaton field [45], and baryogenesis in large extra dimension models [46]. On the other hand, the $\mathrm{RH}$ sneutrino carries lepton number and its decay to light (s)leptons violates lepton number also. One should therefore examine whether the generated asymmetry in the condensate survives after the $\tilde{N}$ decay.

The leading decay channels for $\tilde{N}$, read from Eq.(1), are $\tilde{N} \rightarrow H_{u} \tilde{L}$ and $\tilde{N} \rightarrow \overline{\tilde{H}}_{u} \bar{L}$, creating lepton numbers +1 and -1 respectively. In the limit of unbroken supersymmetry both channels have the same decay width $\left(h^{2} / 8 \pi\right) M_{N}$ (ignoring final state masses, which should be $\ll M_{N}$ in the supersymmetric limit). Thus no net asymmetry will be generated among the left-handed (s)leptons, even for maximal asymmetry in the $\tilde{N}$ sector. ${ }^{*}$ This is intuitively understandable as no lepton asymmetry can be produced from the the decay of RH neutrinos (in the absence of $\mathrm{CP}$-violating phases). In the supersymmetric limit one expects that the same also holds for RH sneutrinos.

Supersymmetry breaking is therefore needed for obtaining an asymmetry in $\tilde{N}$ decay. The low-energy soft terms result in different partial widths for $\tilde{N}$ decays into channels with bosonic and fermionic final states. The presence of $A$-terms allows the conjugate two-body de-

A sufficiently large mass for the $H_{u} L$ flat direction could for example arise in the presence of sizable Hubble-induced gaugino masses and/or Hubble-induced $\mu$ term.

*One might wonder that an asymmetry is produced in the three-body decay channel derived from the four-point vertex $\tilde{N} \tilde{L}_{\tilde{Q}} \tilde{t}$, where $\tilde{Q}_{3}$ and $\tilde{t}$ are the top squark doublet and singlet respectively. However, there are three other three-body channels mediated by the Higgs boson and Higgsino. Once again, in the supersymmetric limit, the sum of all three-body channels yields no net asymmetry [36]. 
cay mode $\tilde{N} \rightarrow \bar{H}_{u} \overline{\tilde{L}}$. Also, due to soft masses, the rates for $\tilde{N} \rightarrow \overline{\tilde{H}}_{u} \bar{L}$ and $\tilde{N} \rightarrow H_{u} \tilde{L}$ channels will be different by an amount of $\sim 2\left(m_{0} / M_{N}\right)^{2}$. The low-energy soft terms therefore yield a lepton asymmetry suppressed by a factor $\left(m_{0} / M_{N}\right)^{2}$ with respect to the initial $\tilde{N}$ asymmetry. This is usually too small since $m_{0} \ll M_{N}$, and hence low-energy supersymmetry breaking alone is not sufficient for successful leptogenesis.

However, supersymmetry is strongly broken in the early Universe. The inflaton energy density, as mentioned earlier, induces a soft mass ${ }^{2}$ of order $H^{2}$ for the scalars. Also, finite temperature effects in the primordial thermal bath give rise to a split in the mass ${ }^{2}$ of scalars and fermions $\propto T^{2}$. We are interested in supersymmetry breaking at the time of $\tilde{N}$ decay, i.e. when $H \simeq$ $\Gamma_{N} \ll M_{N}$. At this time Hubble-induced supersymmetry breaking is already quite small. On the other hand, thermal (supersymmetry breaking) mass corrections can be substantial since $T \simeq M_{N}$ is possible. As mentioned earlier, we assume that the Universe is still matterdominated at the time of $\tilde{N}$ decay. However, for $H>\Gamma_{\phi}$ the exponential decay of the inflaton results in a thermal bath with temperature $T=1.4\left(H T_{\mathrm{R}}^{2} M_{\mathrm{P}} g_{*}^{-1 / 2}\right)^{1 / 4}[42]$; here $\Gamma_{\phi}$ is the inflaton decay width, $T_{\mathrm{R}}$ is the reheat temperature (i.e. the temperature of the Universe at the transition from the matter-dominated to the radiationdominated epoch, after most inflatons have decayed), and $g_{*} \simeq 225$ is the number of light degrees of freedom in the MSSM. The maximum temperature of this instantaneous thermal bath $T_{\max }$ depends on the thermalization rate of inflaton decay products (for details on thermalization, see [47]). However, we generally expect that $T \gtrsim M_{N}$ is possible if $T_{\mathrm{R}} \gtrsim M_{N} / 50$.

The thermal masses for the slepton $\tilde{L}$, Higgs $H_{u}$, lepton $L$ and Higgsino $\tilde{H}_{u}$ are [37]:

$$
\begin{array}{r}
m_{H_{u}}^{2}=2 m_{\tilde{H}_{u}}^{2}=\left(\frac{3}{8} g_{2}^{2}+\frac{1}{8} g_{1}^{2}+\frac{3}{4} h_{t}^{2}\right) T^{2} \\
m_{\tilde{L}}^{2}=2 m_{L}^{2}=\left(\frac{3}{8} g_{2}^{2}+\frac{1}{8} g_{1}^{2}\right) T^{2}
\end{array}
$$

where $g_{1}$ and $g_{2}$ are the $U(1)_{Y}$ and $S U(2)$ gauge couplings, respectively, and $h_{t}$ is the top Yukawa coupling. Thermal corrections to the sneutrino mass are $\propto h$, thus negligible. For a detailed study of finite temperature effects on leptogenesis see [12]. At energies of order $M_{N}$ we take $\left(3 g_{2}^{2}+g_{1}^{2}\right) / 8=1 / 6, h_{t}^{2}=1 / 2$. We therefore have

$m_{B}^{2} \equiv m_{H_{u}}^{2}+m_{\tilde{L}}^{2} \simeq 0.71 T^{2} ; m_{F}^{2} \equiv m_{\tilde{H}_{u}}^{2}+m_{L}^{2} \simeq 0.35 T^{2}$.

The precise values of $m_{B, F}$ are not very important; however, the relation $m_{B}^{2}=2 m_{F}^{2}$, which holds independently of the numerical values of the coupling constants, means that fermionic $\tilde{N}$ decays open up first.

A second effect of the thermal bath is due to different statistics of bosons and fermions. Decays into fermionic [bosonic] final states are suppressed [enhanced] by a factor $\left(1-f_{F}\right)^{2}\left[\left(1+f_{B}\right)^{2}\right]$. Here, $f_{F}$ and $f_{B}$ are the fermion and boson occupation numbers, given by

$$
f_{F}(E)=\frac{1}{\mathrm{e}^{E / T}+1} ; f_{B}(E)=\frac{1}{\mathrm{e}^{E / T}-1} .
$$

Altogether the total $\tilde{N}$ decay width in the presence of a thermal bath can thus be written as

$$
\Gamma_{N}=\Gamma_{N}^{B}+\Gamma_{N}^{F} .
$$

The partial width into the bosonic $H_{u} \tilde{L}$ final state is given by

$$
\Gamma_{N}^{B}=\frac{h^{2} M_{N}}{8 \pi} \lambda^{1 / 2}\left(1, \frac{m_{H_{u}}^{2}}{M_{N}^{2}}, \frac{m_{\tilde{L}}^{2}}{M_{N}^{2}}\right)\left(1+f_{B}\right)^{2},
$$

while the partial width into the fermionic $\overline{\tilde{H}}_{u} \bar{L}$ final state is

$$
\Gamma_{N}^{F}=\frac{h^{2} M_{N}}{8 \pi} \lambda^{1 / 2}\left(1, \frac{m_{\tilde{H}_{u}}^{2}}{M_{N}^{2}}, \frac{m_{L}^{2}}{M_{N}^{2}}\right)\left(1-f_{F}\right)^{2} .
$$

Here, the kinematical factor is given by

$$
\lambda^{1 / 2}(1, a, b)=\sqrt{(1-a-b)^{2}-4 a b},
$$

and the functions $f_{B}, f_{F}$ in Eqs.(18) and (19) should be taken at $E=M_{N} / 2$. Recall that the bosonic (fermionic) final state carries lepton number $+1(-1)$. The efficiency with which a lepton asymmetry can be transferred from the sneutrino condensate to light (s)leptons is thus described by the quantity

$$
\Delta_{B F} \equiv \frac{\Gamma_{N}^{B}-\Gamma_{N}^{F}}{\Gamma_{N}^{B}+\Gamma_{N}^{F}} .
$$

The evolution of the lepton number density carried by the light $\tilde{N}$ decay products, $L_{D}$, is therefore described by the first-order differential equation

$$
\dot{L}_{D}+3 H L_{D}=L_{\tilde{N}} \Gamma_{N} \Delta_{B F},
$$

where $L_{\tilde{N}}$ has been defined in Eq.(10).

Let us discuss the behavior of $\Delta_{B F}$. At $T>1.2 M_{N}$, thermal effects block all $\tilde{N}$ decays, i.e. $\Gamma_{N}=0$. Eqs.(15) show that the leptonic mode opens up first, i.e. for $1.2 M_{N}>T>0.9 M_{N}$, we have $\Gamma_{N}=\Gamma_{N}^{F}$, which implies $\Delta_{B F}=-1$. For $T<0.9 M_{N}$, the bosonic mode also becomes allowed, and quickly takes over owing to the rather strong statistical effects at $T \sim M_{N}$, see Eqs.(16). At somewhat lower temperatures an expansion in $T / M_{N}$ becomes possible; one finds

$$
\Delta_{B F} \simeq-\frac{0.18 T^{2}}{M_{N}^{2}}+2 \exp \left(-\frac{M_{N}}{2 T}\right) .
$$

The second term is dominant when $T>M_{N} / 15$ but drops very quickly for smaller $T$, where the first term 
takes over, i.e. $\Delta_{B F}$ changes sign for a second time; however, at this point $\left|\Delta_{B F}\right| \lesssim 10^{-3}$ is already too small for an efficient transfer of the lepton asymmetry from the $\tilde{N}$ condensate to the $\tilde{N}$ decay products.

In order to find the final baryon asymmetry we again focus on the case $\Gamma_{N}>\Gamma_{\phi}$. In fact, this will be necessary for successful leptogenesis when $|B| \simeq m_{0}$. The reason is that $\Gamma_{\phi}>\Gamma_{N} \simeq m_{0}$ will result in $T_{\mathrm{R}} \sim \sqrt{\Gamma_{\phi} M_{\mathrm{P}}}>$ $10^{10} \mathrm{GeV}$, thus leading to gravitino overproduction ${ }^{\dagger}$. At $H=M_{N}$, we have $\rho_{\phi}=3 M_{N}^{2} M_{\mathrm{P}}^{2}$. For $\Gamma_{\phi}<H<m_{\phi}$ the Universe is matter-dominated and the inflaton number density is redshifted $\propto R^{-3}$, where $R \propto t^{2 / 3}$ is the scale factor of the Universe; this is nothing but the $1 / t^{2}$ factor of Eqs.(11) and (13):

$$
n_{\phi}(t)=\frac{\rho_{\phi}}{m_{\phi}}=\frac{3 H^{2} M_{\mathrm{P}}^{2}}{m_{\phi}}=\frac{4 M_{\mathrm{P}}^{2}}{3 t^{2} m_{\phi}} .
$$

Eventually the inflatons will decay, thereby reheating the universe, i.e. releasing a large amount of entropy:

$$
\frac{n_{\phi}\left(t=\Gamma_{\phi}^{-1}\right)}{s} \simeq \frac{3 T_{\mathrm{R}}}{4 m_{\phi}},
$$

where $s$ is the entropy density. Note that for $\Gamma_{\phi}<H<$ $\Gamma_{N}$ the ratio $L_{D} / n_{\phi}$ will remain constant. We saw at the beginning of Sec. I that the lepton to baryon conversion efficiency through sphaleron effects is about $1 / 3$. Altogether we thus have as final asymmetry

$$
\frac{n_{B}}{s} \simeq \frac{1}{3} \cdot \frac{L_{D}(t)}{n_{\phi}(t)} \cdot \frac{n_{\phi}\left(t=\Gamma_{\phi}^{-1}\right)}{s},
$$

where the quantities in the second factor can be taken at any time $t \gg \Gamma_{N}^{-1}$. Note that the inflaton mass $m_{\phi}$ cancels between the second and the third factor in Eq.(26).

In general $L_{D}$ in Eq.(26) has to be computed by numerically solving Eqs.(6) and (22). However, in order to get a rough estimate of the final asymmetry we can use the approximate result (13) for $L_{\tilde{N}}$, and assume that it is transferred to $L_{D}$ with efficiency $\Delta_{B F}$. Moreover, we can estimate the initial energy density in the sneutrino condensate as $\rho_{\tilde{N}} \simeq M_{N}^{2} N_{0}^{2}$. For $m_{\phi}>H>\Gamma_{N}$ we then have

$$
\frac{n_{\tilde{N}}}{n_{\phi}} \simeq \frac{m_{\phi} N_{0}^{2}}{3 M_{N} M_{\mathrm{P}}^{2}} .
$$

Together with Eq.(25) and again including the 1/3 efficiency for lepton to baryon conversion, this leads to the estimate

\footnotetext{
${ }^{\dagger}$ If $\tilde{N}$ dominates the energy density of the Universe, its decay can dilute the excess of gravitinos. In that case $\tilde{N}$ can act as the curvaton and be responsible for cosmological density perturbations $[31,48,49]$. We will come back to his possibility in Sec. VI F.
}

$$
\frac{\left|n_{B}\right|}{s} \lesssim \frac{T_{\mathrm{R}} N_{0}^{2}}{12 M_{N} M_{\mathrm{P}}^{2}}\left|\Delta_{B F}\right|\left|\sin 2 \theta_{0}\right| .
$$

Here $\Delta_{B F}$ should be computed at temperature $T \simeq$ $\left(g_{*}^{-1 / 2} \Gamma_{N} T_{\mathrm{R}}^{2} M_{\mathrm{P}}\right)^{1 / 4}$, which is the temperature of the thermal plasma at $H=\Gamma_{N}$. The (approximate) equality holds if (most) $\tilde{N}$ decays occur while $\left|t^{2} L_{\tilde{N}}\right|$ is near its maximum; we saw earlier that this requires $\Gamma_{N} \simeq|B|$ if the Hubble-induced contribution to the $B$-parameter is negligible.

\section{CONSTRAINTS}

So far we have assumed that the sneutrino condensate starts oscillating when $H \simeq M_{N}$, and survives until $H \simeq \Gamma_{N}$, at which point it decays via perturbative one-particle decays. However, neither the survival of the condensate as a coherent state*, nor its perturbative decay is in general guaranteed. Here we show that these requirements can be translated into an upper and lower bound on the reheat temperature $T_{\mathrm{R}}$, respectively. These constraints apply equally to the scenario of refs. [30,31], which also rely on the perturbative decay of an $\tilde{N}$ condensate.

\section{A. Resonant decay of the condensate}

The decay of coherent oscillations of a massive scalar field in cosmology was initially considered in the context of inflation [50]. The energy-momentum tensor of such an oscillating field, when averaged over oscillations, resembles that of non-relativistic matter with the same mass. If the decay occurs over many oscillations, which is presumably the case for sufficiently weak couplings, its rate should be the same as the one-particle decay rate of non-relativistic matter. However, the situation can be more complicated for larger couplings. Due to the coherent nature of oscillations, the occupation number of decay products can become large in which case the one-particle decay approximation will not be adequate. This was noticed rather recently in the case of inflaton decay [51,52]. In fact, depending on the amplitude of oscillations and the size of its coupling, the condensate decay can occur in different regimes (typically non-perturbatively) with different outcomes. Recently,

\footnotetext{
*We do not expect the condensate to fragment into $Q$-balls [34]. This could happen if the potential increased slower than quadratically at large field values, e.g. if RG evolution decreased the relevant mass. However, in our case the RG evolution of $M_{N}$ is negligible, since $N$ is a gauge singlet with very small Yukawa couplings. Nevertheless, the condensate could be destroyed by thermal effects.
} 
the possibility of non-perturbative decay of an oscillating $\tilde{N}$ condensate has also been discussed [53]. Here we briefly go through different regimes for $\tilde{N}$ decay, point out implications of a non-perturbative decay for the lepton asymmetry, and discuss effects which can shut it off.

We shall focus on the decay of the $\tilde{N}$ condensate to scalars as it provides the most efficient channel for energy transfer. The dominant terms responsible for such decays are derived from the superpotential in (1)

$$
h^{2}|\tilde{N}|^{2}\left[\left|H_{u}\right|^{2}+|\tilde{L}|^{2}\right]+\left(h M_{N} \tilde{N}^{*} H_{u} \tilde{L}+\text { h.c. }\right) \text {. }
$$

First consider the case when $\hat{N} \gg M_{N} / h, \hat{N}$ being the amplitude of $\tilde{N}$ oscillations; initially we have $\hat{N}(H=$ $\left.M_{N}\right)=N_{0}$, see Eq.(5). In this regime the first two terms in Eq. (29) are dominant leading to explosive production of $H_{u}$ and $\tilde{L}$ quanta with (physical) energies $\lesssim\left(h \hat{N} M_{N}\right)^{1 / 2} \gg M_{N}$. This process, called preheating, typically completes in a time scale of several tens of $M_{N}^{-1}$ [51]. Eventually, due to re-scatterings, one has a plasma of $\tilde{N}, H_{u}$ and $\tilde{L}$ quanta with large occupation numbers and typical momenta $\lesssim\left(h \hat{N} M_{N}\right)^{1 / 2}$. The preheat plasma (and every other field which is coupled to it) is in kinetic equilibrium, but full thermal equilibrium takes much longer to establish [54]. It is noticeable that particles with a mass up to $\left(h \hat{N} M_{N}\right)^{1 / 2}$ can be produced during preheating.

The amplitude $\hat{N}$ is redshifted $\propto 1 / t$ if preheating is blocked [see Eq.(30) below]. Eventually we have $\hat{N} \ll$ $M_{N} / h$, where the cubic terms in Eq. (29) will be dominant. If $M_{N}^{2} /\left(h^{2} N_{0}\right) \ll \hat{N} \ll M_{N} / h$, the condensate decay occurs in the narrow-band resonance regime [51,52]; note that the first of these strong inequalities requires $N_{0} \gg M_{N} / h$, since $\hat{N} \leq N_{0}$. In this regime $H_{u}$ and $\tilde{L}$ quanta with occupation numbers larger than one and energy narrowly peaked around $M_{N} / 2$ are produced, at a rate given in [52]. If $\hat{N} \ll M_{N}^{2} /\left(h^{2} N_{0}\right)$, the decay is perturbative and its rate will be given by the familiar one-particle decay rate. Finally, if $h \hat{N} \simeq M_{N}$, all terms in (29) are comparable. In this case the cubic terms result in a tachyonic instability during part of oscillations which can again lead to a rapid decay of $\tilde{N}$ [53].

The decay of $\tilde{N}$ oscillations via preheating has undesirable consequences for successful leptogenesis. First, $\Gamma_{\text {preheat }} \gg|B|$, and hence the asymmetry in the condensate, given in Eq. (10), is suppressed. Moreover, since particles in the preheat plasma have energies $\gg M_{N}$, lepton number violating processes mediated by $\tilde{N}$ and $N$ will be efficient. Therefore any existing lepton asymmetry will quickly be washed away ${ }^{\dagger}$. The situation is some-

\footnotetext{
${ }^{\dagger}$ Since preheating destroys the coherence of $\tilde{N}$ oscillations, the $B$-term will not generate a (large) net asymmetry from the preheat plasma.
}

what better for narrow-band resonance decay. There are two effects competing with each other in this case. On the one hand, $\Gamma_{\text {narrow }}>|B|$ which suppresses the asymmetry in the condensate unless the Hubble $B$-term is sizeable. On the other hand, $\Delta_{B F} \simeq 1$ as the condensate essentially decays to scalars. Therefore the yielded lepton asymmetry can actually be larger than that in a perturbative decay, unless $\Gamma_{\text {narrow }} \gg|B|$.

The picture given above is for a simple toy model. In realistic models resonant decay can be altered, suppressed or postponed due to various effects. For example, final state self-couplings of moderate strength can qualitatively alter resonant decay $[55,56]$. Also, for a complex oscillating field, preheating can be postponed due to out-of-phase oscillations of the real and imaginary components [57]. In the case of sneutrino both of these effects are present. The decay products $H_{u}$ and $\tilde{L}$ have $D$-term couplings of gauge strength to each other, and the $B$-term causes out-of-phase oscillations of $\tilde{N}_{R}$ and $\tilde{N}_{I}$. These, as pointed out in [53], can regulate the resonant decay of $\tilde{N}$ oscillations.

In the absence of a complete understanding of possible non-perturbative decays of the $\tilde{N}$ condensate, it is safer to require that these non-perturbative effects are suppressed. One possibility is to require that $h N_{0}<M_{N}$. However, we will see in Sec. $\mathrm{V}$ that this results in too small a baryon asymmetry. The second possibility is to prevent resonant decays until $\hat{N}$ is redshifted to a sufficiently small value. This will in fact happen if the medium is sufficiently hot. We saw that preheating can produce particles with mass up to $\left(h N_{0} M_{N}\right)^{1 / 2}$. Together with Eqs.(15) this implies that thermal effects can kinematically shut off preheating if $T_{0}^{2}>h N_{0} M_{N}$, which requires

$$
T_{\mathrm{R}}^{2} M_{\mathrm{P}}>\frac{\sqrt{g_{*}}}{4} h^{2} N_{0}^{2} M_{N}
$$

Since (in the absence of preheating) the oscillation amplitude scales like $1 / t$, while $T^{2} \propto 1 / \sqrt{t}$, the constraint (30) also forbids non-perturbative $\tilde{N}$ decay, including decays in the narrow-band resonance regime, at all later times. It is therefore a sufficient condition for ensuring that the sneutrino condensate decays perturbatively.

We want these perturbative decays to occur when $H \simeq$ $h^{2} M_{N} /(4 \pi)$. We saw in the discussion of Eq.(22) that this requires $T<1.2 M_{N}$ at that time, which implies

$$
\frac{h^{2}}{4 \pi} T_{\mathrm{R}}^{2} M_{\mathrm{P}}<M_{N}^{3} \sqrt{g_{*}} .
$$

Otherwise thermal corrections postpone the perturbative decay $[55,58]$. This may actually be desired, if $|B|<h^{2} M_{N} /(4 \pi)$. However, we will see below that the constraint (31) has to be satisfied if coherent oscillations of the condensate are to continue until $H \simeq \Gamma_{N}$. 


\section{B. Thermal destruction of the condensate}

One has to also ensure that the coherence of oscillations is not lost by scatterings off particles in the thermal bath [59]. The most efficient reactions are $\tilde{N}$ annihilation with $H_{u}$ and $\tilde{H}_{u}, L$ and $\tilde{L}$, top (s)quarks and right-handed bottom (s)quarks; the corresponding matrix elements are $\propto h h_{t}^{\ddagger}$. Coherence will be lost if the rate for such reactions is faster than the expansion rate $H$. We therefore have to require

$$
\frac{63 \xi(3) h^{2} h_{t}^{2}}{16 \pi^{3}} \cdot \frac{T^{3}}{M_{N}^{2}+6 T M_{N}}<H .
$$

The factor 63 counts the final state multiplicities and number of initial state degrees of freedom (which enter the target density); the product of these two coefficients is in fact identical for all bosonic reactions. We have included a factor 1.75 to take annihilation on a fermion into account. The factor $T^{3}$ also comes from the density of particles in the plasma, and $M_{N}^{2}+6 T M_{N}$ is a typical squared center-of-mass energy $\left(\right.$ at $\left.T<M_{N}\right)$. The condition (32) has to be satisfied at all times between the onset of oscillations $\left(H \simeq M_{N}\right)$ until their perturbative decay $\left[H \simeq h^{2} M_{N} /(4 \pi)\right]$. In the matter-dominated era, $H \propto T^{4}$. Condition (32) is therefore most difficult to satisfy at the latest relevant time. Conservatively ignoring the $6 T M_{N}$ term in the denominator, and setting $h_{t}^{2}=0.5$ and $T=1.4\left(T_{R}^{2} H M_{P} g_{*}^{-1 / 2}\right)^{1 / 4}$ as in Sec. II, we find as relevant constraint at $H=h^{2} M_{N} /(4 \pi)$ :

$$
\frac{h^{2}}{4 \pi} T_{\mathrm{R}}^{2} M_{P}<\frac{\sqrt{g_{*}}}{4} M_{N}^{3}
$$

This bound is very similar to, although slightly stronger than, the condition (31) above, i.e. it implies that thermal effects do not delay the perturbative decay of the $\tilde{N}$ condensate significantly.

Moreover, it is also possible that thermal effects trigger early oscillations of $\tilde{N}$ [59]. This will happen, provided that $h N_{0}<T$ (so that Higgs/Higgsino and (s)leptons will be in thermal equilibrium) and $h T>H$ (so that they induced a thermal mass for $\tilde{N}$ exceeding the Hubble rate) when $H>M_{N}$. Early oscillations dampen $\tilde{N}$ more than the case with $H_{o s c} \simeq M_{N}$, thus leading to a smaller lepton asymmetry. Also, so long as $h T>M_{N}$,

\footnotetext{
${ }^{\ddagger}$ There are also annihilation diagrams including electroweak gauge and gaugino fields. These have a smaller multiplicity factor, and are hence subdominant. In addition, $2 \rightarrow 1$ annihilations with (s)leptons into an on-shell $H_{u}$ or $\tilde{H}_{u}$, which have a larger phase space factor, can also be active. These, however, are kinematically allowed when $T$ is sufficiently higher than $M_{N}$. Therefore, since $H \propto T^{4}$, they can be safely neglected.
}

the thermal mass of the sneutrino $h T$ replaces $M_{N}$ in all calculations. However, $h T<M_{N}$ at the time of sneutrino decay since otherwise the large thermal mass of final states kinematically blocks the decay. Therefore the condition for preventing evaporation of the condensate is still given by (33). This condition implies that $h T>M_{N}$ is only possible at $H>M_{N} /\left(4 \pi h^{2}\right)$. If the constraint in (33) is saturated, $h T=M_{N}$ implies $H=M_{N} /\left(4 \pi h^{2}\right)$; this exceeds $h T$ by the (large) factor $1 /\left(4 \pi h^{2}\right)$. The mismatch between thermal mass and Hubble damping becomes even worse at earlier times, since $H \propto T^{4}$. We thus conclude that the condensate will not undergo early oscillations.

\section{NUMERICAL RESULTS}

We are now ready to present numerical results. For the reasons given in the previous Section, we focus on scenarios where the $\mathrm{RH}$ sneutrino condensate decays through the perturbative single--particle decay discussed in Sec. III, i.e. we impose the lower and upper bounds (30) and (33), respectively, on the reheat temperature. The baryon asymmetry is computed by solving the equations of motion (6) for $\tilde{N}$ as discussed in Sec. II, and using the lepton asymmetry in the sneutrino condensate obtained from this solution via Eq.(10) as input in Eq.(22).* The numerical solution of this equation [with initial condition $L_{D}\left(H=M_{N}\right)=0$ ] gives the lepton number density, which is translated in the final baryon-to-entropy ratio using Eq.(26).

In Figs. 1a,b we show the evolution of the temperature and of the lepton number stored in the $\tilde{N}$ condensate and its decay products for two scenarios with rather heavy right-handed neutrinos $\left(M_{N}=3 \cdot 10^{10} \mathrm{GeV}\right)$ and high reheat temperature $\left(T_{\mathrm{R}}=10^{9} \mathrm{GeV}\right)$. The first scenario has sizable Hubble-induced soft SUSY breaking $b=0.1$. As a result the lepton asymmetry in the condensate (dashed, blue curve) grows almost monotonically with time until $t \simeq \Gamma_{N}^{-1}$; the wiggles at early time are related to the terms $\propto \Delta$ in Eq.(11) (as well as to terms not included in this approximate analytical solution). The lepton asymmetry of the decay products (solid, red curve) remains zero until $T=1.2 M_{N}$, since for higher temperatures thermal effects forbid perturbative $\tilde{N}$ decays. [Since the condition (30) is satisfied, non-perturbative decays of the condensate are forbidden as well.] Then the fermionic $\tilde{N}$ modes open up, leading to a negative lepton number carried

\footnotetext{
* It is more convenient to numerically solve the equations of motion for the "co-moving" fields $t N_{R, I}(t)$ as well as for the co-moving lepton density $t^{2} L_{D}(t)$. As shown in Sec. II, the former can to good approximation be solved analytically by Eq.(7) if the Hubble-induced supersymmetry breaking is weak, which is always true at sufficiently late time.
} 

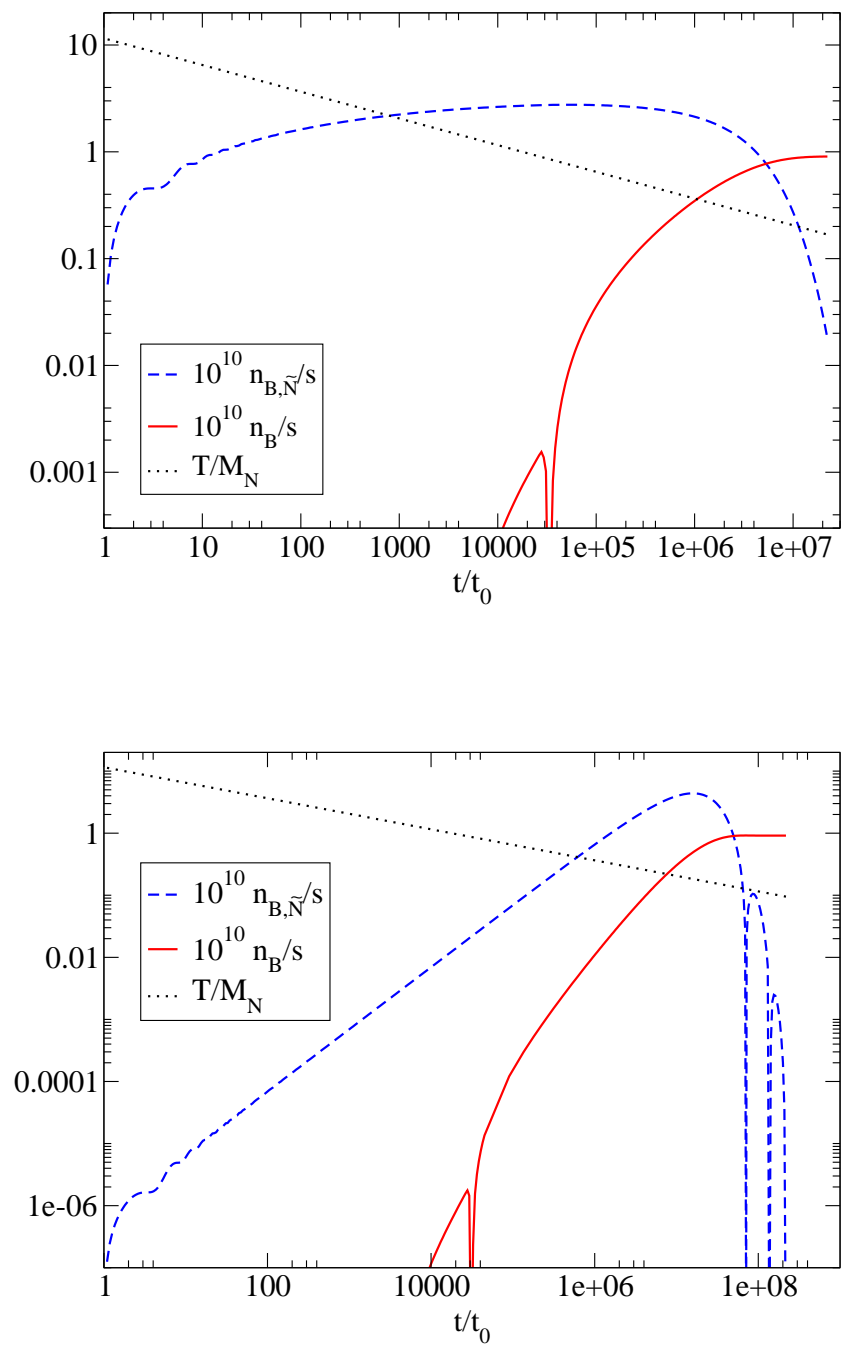

FIG. 1. Time evolution of the temperature in units of $M_{N}$ (dotted, black), and the absolute values of the lepton number in the RH sneutrino condensate (dashed, blue), and in the $\tilde{N}$ decay products (solid, red). The lepton numbers are normalized using Eq.(26), i.e. we show the baryon-to-entropy ratio that would result if the lepton number present at time $t$ was converted into a baryon asymmetry via sphalerons. The parameters in the upper (lower) figure correspond to those in the first (second) row of Table 1. We have imposed the initial condition (5) at $H=M_{N}$, i.e. $t_{0}=2 /\left(3 M_{N}\right)$. by the decay products. At $T=0.9 M_{N}$ bosonic $\tilde{N}$ decays become possible, and quickly take over because of the Bose enhancement. The lepton asymmetry of the decay products therefore changes sign, and thereafter grows monotonically. Note that $L_{\tilde{N}}$ remains almost constant for quite a long time. Eventually the Hubble-induced $B$-term becomes subdominant to the soft term, taken as $B=100 \mathrm{GeV}$ in this example, and $L_{\tilde{N}}$ starts to oscillate. However, for the given choice $h=0.002$ most of the condensate has already decayed by that time, so these oscillations essentially have no effect on the final baryon asymmetry.

The second scenario is for negligible Hubble-induced supersymmetry breaking. Although we have increased $B$ to $1 \mathrm{TeV}$, the growth of the asymmetry $L_{\tilde{N}}$ of the condensate is now much slower. Since $T_{\mathrm{R}}$ remains the same, $\tilde{N}$ decays commence at the same time as in the first scenario. Since $L_{\tilde{N}}$ is still quite small at that time, the growth of $L_{D}$ is initially also much slower than in the first scenario; note the different scales for the $y$-axes of the two figures. To compensate for this, a somewhat higher maximal value of $L_{\tilde{N}}$ is required; this has been obtained by adjusting $N_{0}$ accordingly. In other words, scenarios with $b=0$ are somewhat less efficient in transferring the lepton asymmetry from the condensate to its decay products. We also note much more prominent oscillations of $L_{\tilde{N}}$ than in the first case. However, the second, "wrong-sign" peak in $\left|L_{\tilde{N}}\right|$ is suppressed by about a factor of 50 due to $\tilde{N}$ decays, and can therefore not deplete the final baryon asymmetry significantly.

Successful baryogenesis is also possible for significantly smaller values of $M_{N}, T_{\mathrm{R}}$ and $B$. Some examples are shown in Table 1. The first two rows are the two scenarios depicted in Figs. 1. Reducing $M_{N}$ requires that the product $h T_{\mathrm{R}}$ be reduced as well to satisfy the condition (33). At the same time we have to satisfy the lower bound (30) on $T_{\mathrm{R}}$. These two conditions together imply that scenarios with reduced $M_{N}$ are only successful if $h$ and $T_{\mathrm{R}}$ are reduced as well, roughly $\propto \sqrt{M_{N}}$ and $\propto M_{N}$, respectively. The $\tilde{N}$ decay width then scales $\propto M_{N}^{2}$, which requires a corresponding reduction of $|B|$ to maintain the "matching" $|B| \simeq \Gamma_{N}$ required for an effective transfer of the asymmetry from the condensate to the decay products. The approximate result (28) indicates that the final baryon asymmetry will be unchanged if the ratio $M_{N} / T_{\mathrm{R}}$ remains constant, and indeed we see that the required initial field value $N_{0}$ does not change too much in the successful scenarios shown in the Table. Note that this value is somewhat below the scale of Grand Unification $M_{G U T} \simeq 2 \cdot 10^{16} \mathrm{GeV}$. This is compatible with $N$ being charged under the GUT gauge group, in which case the new $D$-term limits $\left|N_{0}\right| \lesssim M_{G U T}$ as discussed earlier.

It is also necessary that the "inverse decay" of $\tilde{N}$ does not erase the generated lepton asymmetry; that is, lepton number violating processes where a (real or virtual) $\tilde{N}$ (or $N$ ) is exchanged must not be in equilibrium. For 
TABLE I. Some examples of parameters which lead to a successful soft leptogenesis from the $\tilde{N}$ condensate for $\sin 2 \theta_{0} \sim 1$ and respect the constraints (30) and (33) on $T_{\mathrm{R}}$. All dimensionful parameters are in $\mathrm{GeV}$.

\begin{tabular}{|c|c|c|c||c|c|}
\hline \hline$B$ & $b$ & $M_{N}$ & $T_{\mathrm{R}}$ & $N_{0}$ & $h$ \\
\hline \hline $10^{2}$ & 0.1 & $3 \cdot 10^{10}$ & $10^{9}$ & $5 \cdot 10^{14}$ & $2 \cdot 10^{-3}$ \\
\hline $10^{3}$ & 0 & $3 \cdot 10^{10}$ & $10^{9}$ & $1.2 \cdot 10^{15}$ & $10^{-3}$ \\
\hline 1 & 0 & $10^{9}$ & $3 \cdot 10^{7}$ & $2 \cdot 10^{15}$ & $10^{-4}$ \\
\hline $10^{-2}$ & 0 & $10^{8}$ & $4 \cdot 10^{6}$ & $1.5 \cdot 10^{15}$ & $3 \cdot 10^{-5}$ \\
\hline $10^{-4}$ & 0 & $10^{7}$ & $10^{6}$ & $5 \cdot 10^{14}$ & $10^{-5}$ \\
\hline $10^{-6}$ & 0 & $3 \cdot 10^{5}$ & $10^{4}$ & $7 \cdot 10^{14}$ & $10^{-5}$ \\
\hline \hline
\end{tabular}

$T \ll M_{N}$, these processes can only proceed via the exchange of a far off-shell $\tilde{N}$ or $N$, and occur at a rate $\propto h^{4} T^{3} / M_{N}^{2}$. The upper bounds (33) and (30) on $h$ imply that these reactions will not be in equilibrium. On the other hand, Figs. 1 indicate that the most relevant temperature for creating the lepton asymmetry in the decay products is not too far below $M_{N}$; indeed, $\left|\Delta_{B F}\right|$ becomes too small for our scenario to be workable (given the other constraints) if the condensate decays at a temperature $T \ll M_{N}$. The creation of (nearly) on-shell $\tilde{N}$ particles from the thermal plasma is then at most mildly Boltzmann-suppressed. However, due to the very small width of $\tilde{N}, 2 \rightarrow 1$ processes (the $N$ inverse decay in the stricter sense) will be kinematically possible only for a very small fraction of all collisions of light particles in the plasma. We estimate the rate for these processes at $T \gtrsim M_{N}$ as

$$
\Gamma_{\mathrm{inv}} \sim \frac{\Gamma_{0}}{4} \frac{2 T}{M_{N}} \int_{M_{N}^{2} /\left(4 T^{2}\right)}^{\infty} \frac{x d x}{e^{x} \pm 1}
$$

where the $+(-)$ sign is for fermionic (bosonic) initial states. Figs. 1 show that most of the asymmetry in the decay products is created at $T \lesssim M_{N} / 3$. According to Eq.(34) this implies $\Gamma_{\text {inv }} \lesssim \Gamma_{N} / 5$, which should be compared with the Hubble expansion rate at $H \simeq \Gamma_{N}$. We therefore expect at worst a small dilution of the asymmetry through inverse $\tilde{N}$ decays, which can easily be compensated by a small increase of $N_{0}$. Higher order $(2 \rightarrow 2)$ scattering reactions producing on-shell $\tilde{N}$ particles are possible for any configuration with cms energy $\geq M_{N}$ (as opposed to almost exactly equal to $M_{N}$ in case of $2 \rightarrow 1$ reactions), but are suppressed by an additional coupling and additional $\pi$ factors from phase space integration; they should thus be subdominant to inverse $\tilde{N}$ decay.

We just saw that successful baryogenesis requires quite small values of the soft breaking parameter $B$ if $M_{N} \lesssim 10^{9} \mathrm{GeV}$. In models of gravity-mediated supersymmetry breaking, the exact value of $B$ depends on the details of supersymmetry breaking sector and the structure of the Kähler potential. For example, in the Polonyi model and with minimal kinetic terms one ob- tains $B=(2-\sqrt{3}) m_{0}[38]$. Under general circumstances one can expect that $|B| \sim m_{0}$. However, for a non-minimal Kähler potential it is possible to have $|B| \ll m_{0}^{\dagger}$. Similarly, simple models of gauge-mediated supersymmetry breaking have $B=0$ at the messenger scale. However, even if $B=0$ at the tree-level, it is inevitably generated at the one-loop level through the $A$-term associated with the neutrino Yukawa coupling $\ddagger$, and hence in general $B$ will not remain strictly zero. Note that these loop corrections are $\mathcal{O}\left(h^{2}\right)$, so any value of $m_{0} \gtrsim|B| \gtrsim h^{2} m_{0}$ is (technically) natural. The values of $B$ used in Table 1 fall in this range if the scale of (most) soft breaking parameters $m_{0} \sim 1 \mathrm{TeV}$.

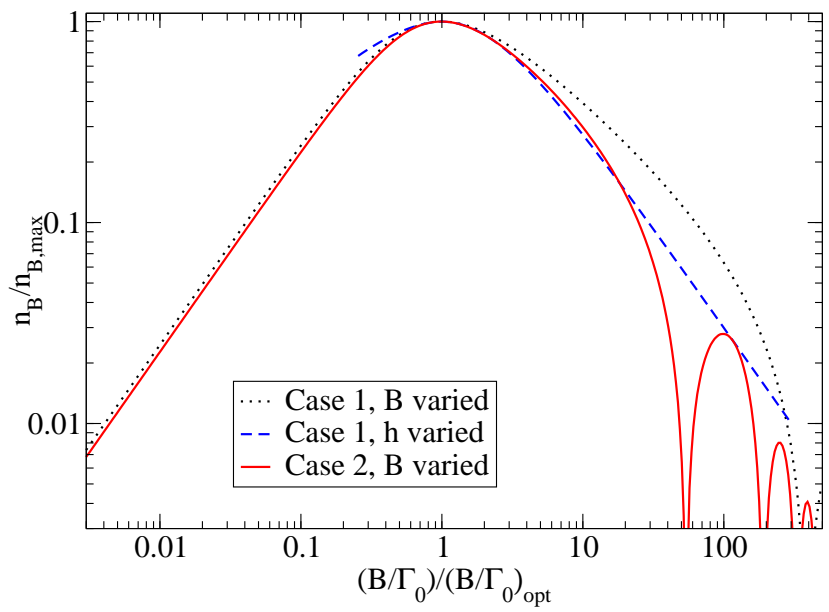

FIG. 2. Dependence of the final baryon asymmetry on the deviation of the ratio $B / \Gamma_{0}$ from its optimal value, which is near $1 ; \Gamma_{0}=h^{2} M_{N} /(4 \pi)$ is the zero-temperature width of the RH sneutrino $\tilde{N}$. Dotted (black) and solid (red) curves correspond to the second and last scenario of Table 1, except that $B$ has been varied. The dashed (blue) curve again corresponds to the second line of Table 1 , but now the coupling $h$ has been varied.

The results of the Table confirm that Eq.(28) provides a reasonable approximation if the Hubble- $b$ can be ne-

\footnotetext{
${ }^{\dagger}$ Non-minimal Kähler terms for $\tilde{N}$ may be preferred by other considerations. For example, it is required for the flatness of the potential at $|\langle\tilde{N}\rangle|>M_{\mathrm{P}}$, if $\tilde{N}$ is the inflaton [60].

${ }^{\ddagger}$ If this $A$-term itself vanishes at tree-level, it will be generated through gauge interactions from the electroweak gaugino masses. If $\mathbf{N}$ carries some gauge charge, $B$ will also be generated at one-loop through gauge interactions.

${ }^{\S}$ If $N$ carries some gauge charge, and the messenger scale is larger than the scale where the corresponding gauge symmetry is broken, the natural lower bound on $|B|$ increased to $\sim m_{1 / 2} /\left(16 \pi^{2}\right)$, where $m_{1 / 2} \gtrsim 100 \mathrm{GeV}$ is a gaugino mass; this excludes scenarios with $|B| \ll 1 \mathrm{GeV}$.
} 
glected and the resonance condition $\Gamma_{N} \simeq|B|$ is satisfied, with $\Delta_{B F} \simeq 0.05$ to 0.25 . Fig. 2 shows how the final baryon asymmetry is reduced when we deviate from this relation. We see that the asymmetry has a clear maximum when plotted against the ratio $B / \Gamma_{0}$, where $\Gamma_{0}$ is the width of $\tilde{N}$ at temperature $T=0$. However, the curve is rather asymmetric around its maximum. If $|B|$ is reduced from its optimal choice, leaving $h$ (and hence $\Gamma_{0}$ ) unchanged, one finds an almost universal behavior once both the asymmetry and the ratio $|B| / \Gamma_{0}$ are normalized to their values at the maximum of $n_{B} / s$; for $|B|$ well below the optimal choice, the asymmetry becomes simply $\propto|B|$. This can be understood from the fact that the lepton number of the condensate at time $t \simeq 1 / \Gamma_{N}$ is $\propto|B|$ for small $|B|$, see Eq.(10).

On the other hand, different scenarios give different results when $|B|$ is increased beyond its optimal choice. The crucial variable here is the temperature of the plasma at time $t \simeq 1 / \Gamma_{N}$, divided by $M_{N}$. This quantity is nearly two times bigger in "Case 2" of Fig. 2 (corresponding to the last row of the Table) than for "Case 1" (the second row of the Table). A smaller temperature means that the quantity $\left|\Delta_{B F}\right|$ will decrease faster with time, see Eq.(23). Hence the final baryon asymmetry remains dominated by the contribution from the first oscillation of the lepton number $L_{\tilde{N}}$ of the condensate, even if only a rather small fraction of the condensate decays during that time; however, eventually the oscillations of $L_{\tilde{N}}$ will manifest itself in the final baryon asymmetry as well. On the other hand, increasing the temperature increases the importance of subsequent minima and maxima of $L_{\tilde{N}}$, thereby leading to earlier oscillations of the final baryon asymmetry (as function of $B$, not as function of time), as shown by the solid curve.

The final asymmetry also depends on whether $|B|$ or $h$ (and hence $\Gamma_{N}$ ) is varied. This can be seen by comparing the dashed and dotted curves in Fig. 2. Reducing $h$ also increases $|B| / \Gamma_{N}$, i.e. the condensate decays "too late". However, in addition to having to average over several oscillations of $L_{\tilde{N}}$, a reduction of $h$ for fixed $T_{\mathrm{R}}$ also implies that the temperature at $t \simeq 1 / \Gamma_{N}$ is reduced; recall that $\left|\Delta_{B F}\right|$ depends exponentially on this ratio, see Eq.(23). A reduction of $h$ therefore first leads to a faster decrease of the baryon asymmetry than an increase of $|B|$ does; conversely, an increase of $h$ beyond the optimal choice leads to a smaller reduction of $n_{B} / s$ than an increase of $|B|$ does. However, we just saw that reducing the temperature at $\tilde{N}$ decay also reduces the importance of the minima (and later maxima) of $L_{\tilde{N}}$. One therefore does not find oscillatory behavior of $n_{B} / s$ when plotted as function of $h$. A final difference is that $B$ can be varied arbitrarily without violating any consistency conditions. In contrast, for fixed values of the other parameters, $h$ is bounded from above by the requirement (33) that the condensate should not be destroyed by scattering off the thermal plasma, as well as by the requirement (30) that non-perturbative $\tilde{N}$ decays be shut off thermally; the latter requirement is the more stringent one in Fig. 2. $\underset{\tilde{N}}{h}$ is also bounded from below, by the requirement that $\tilde{N}$ decays before the inflaton does.

\section{DISCUSSION}

In this section we discuss the merits and consequences of our scenario, compare it with similar suggestions in the literature, and discuss what happens when some of our assumptions are relaxed.

\section{A. Consequences for low-energy physics}

The main particle physics motivation for considering heavy RH neutrinos is undoubtedly the see-saw mechanism [7], where the masses of the light neutrinos come out quadratic in their Yukawa coupling, and inverse to the masses of the heavy neutrinos. Our mechanism will work for a single RH neutrino superfield, which may not be the lightest one. Therefore the effective neutrino mass** $\bar{m}=h^{2} v_{u}^{2} / M_{N}$, where $v_{u}=\left\langle H_{U}^{0}\right\rangle \sim 150 \mathrm{GeV}$, is even more difficult to interpret than in usual leptogenesis models, where only the lightest RH (s)neutrino is relevant. It nevertheless seems reasonable to require

$$
\bar{m} \lesssim 1 \mathrm{eV} \Longrightarrow h \lesssim 10^{-2} \sqrt{\frac{M_{N}}{10^{9} \mathrm{GeV}}}
$$

Otherwise realistic neutrino mass spectra could at best be obtained at the cost of severe cancellations. Note that the entries of Table 1 correspond to significantly smaller values, $\bar{m} \lesssim 0.001 \mathrm{eV}$. Atmospheric neutrino oscillations indicate that at least one neutrino must have a mass $\gtrsim 0.05$ $\mathrm{eV}$ [61]. This indicates that the other RH neutrinos must play an important role in the see-saw mechanism, even though they may be irrelevant for baryogenesis. Since our mechanism is not sensitive to flavor mixing, we cannot sharpen the predictions for (s)lepton flavor violating processes that might be accessible at low energies, compared to "generic" see-saw models [62]. Moreover, in our scenario CP is broken purely spontaneously in the early Universe, through the complex vacuum expectation value of $\tilde{N}$. This obviates the need for any complex phases in the Lagrangian. Hence no prediction can be made for possible $\mathrm{CP}$ violation in neutrino oscillations [63].

\footnotetext{
${ }^{* *}$ In our scenario the coupling $h$ enters only via the total decay width of $\tilde{N}$. The existence of three light generations of (s)leptons can therefore trivially be incorporated by the replacement $h^{2} \rightarrow \sum_{i=1}^{3} h_{i}^{2}$.
} 


\section{B. Comparison with related scenarios}

The fact that our mechanism works for purely real Lagrangian distinguishes it from the proposal of Refs. [36,37], in which the lepton asymmetry is also created with the help of the $B$-term but no condensate exists. There the $\tilde{N}-\tilde{N}^{*}$ asymmetry arises from an absorptive part in the $\tilde{N}-\tilde{N}^{*}$ mixing, and is proportional to the imaginary part of the $A$-term associated with the neutrino Yukawa coupling, denoted by $A_{\nu}$. This results in $[37]$

$$
\frac{L_{D}}{\left(n_{\tilde{N}}+n_{\tilde{N}}\right)} \simeq \frac{4|B| \Gamma_{N}}{4|B|^{2}+\Gamma_{N}^{2}} \frac{\Im \mathrm{m} A_{\nu}}{M_{N}} \Delta_{B F} .
$$

A necessary condition for generating a sufficiently large asymmetry is therefore that the phase of $A_{\nu}$ is large (in the basis where $B$ is real). On the other hand, constraints on the electric dipole moments of the electron and neutron imply that the phases of many other soft breaking terms must be small [64]. This may complicate the construction of complete models where the mechanism of refs. [36,37] is workable. In our scenario the ratio of Eq.(36) is simply given by $\Delta_{B F}$ if $\Gamma_{N}$ and $|B|$ match perfectly; the additional suppression factor due to possible mismatch has been shown in Fig. 2 .

If $\tilde{N}$ has to be produced thermally, the case primarily studied in $[36,37,12]$, the asymmetry in Eq. (36) will be too small when $\left|A_{\nu}\right| \simeq|B| \sim 1 \mathrm{TeV}$. For $M_{N} \leq 10^{9}$ $\mathrm{GeV}$ (to be compatible with the gravitino bound on $T_{\mathrm{R}}$ ) the condition for out-of-equilibrium decay of the sneutrino $\Gamma_{N}<M_{N}^{2} / M_{\mathrm{P}}$ implies that $\Gamma_{N}<1 \mathrm{GeV}$. Thus the first two terms on the $\mathrm{RH}$ side of (36) combine to give a number $<M_{N} / M_{\mathrm{P}}$. The resultant asymmetry, after taking $\Delta_{B F}$ and the entropy factor into account, will be $\ll 10^{-10}$. In fact, it is found in $[37,12]$ that the thermal scenario works only if $|B| \ll 1 \mathrm{TeV}$, for $10^{6} \mathrm{GeV} \lesssim M_{N} \lesssim 2 \cdot 10^{8} \mathrm{GeV}$.

The situation is somewhat better for non-thermal scenarios. For example, consider the case when the (perturbative) inflaton decay to the sneutrino and the subsequent decay of $\tilde{N}$ to light fields reheats the Universe and generates a lepton asymmetry. In this case the $\tilde{N}+\tilde{N}^{*}$ number density (after inflaton decay) is essentially equal to the inflaton number density (before decay). Using Eqs.(36) and (25) we find

$$
\frac{L_{D}}{s} \sim \frac{4|B| \Gamma_{N}}{4|B|^{2}+\Gamma_{N}^{2}} \cdot \frac{\Im \mathrm{m}\left[A_{\nu}\right]}{M_{N}} \Delta_{B F} \cdot \frac{T_{\mathrm{R}}}{m_{\phi}},
$$

where $m_{\phi}>M_{N}$ is the inflaton mass. The product of the first two factors in Eq.(37) is nearly independent of $M_{N}$ so long as $|B| \gtrsim \Gamma_{N}$, since $\Gamma_{N} \propto M_{N}$ for fixed $h$. On the other hand, the see-saw constraint (35) implies that the maximal value of $\Gamma_{N} / M_{N}$ grows $\propto M_{N}$. For $|B| \simeq \Im \mathrm{m}\left[A_{\nu}\right] \geq \Gamma_{N}$ Eq.(37) thus implies

$$
\frac{L_{D}}{s} \sim 10^{-5} \Delta_{B F} \frac{\bar{m}}{1 \mathrm{eV}} \frac{M_{N}}{10^{9} \mathrm{GeV}} \frac{T_{\mathrm{R}}}{m_{\phi}} .
$$

This may allow $M_{N}$ down to $10^{7} \mathrm{GeV}$ if $T_{\mathrm{R}}<M_{N}<m_{\phi}$, and if the bound (35) is nearly saturated. If $M_{N}>10^{9}$ $\mathrm{GeV}$, the bound (35) allows $\Gamma_{N}>1 \mathrm{TeV}$. However, Eq.(37) shows that such scenarios, with $\Gamma_{N}>|B|$ and $M_{N}>10^{9} \mathrm{GeV}$, will generally not yield a sufficient baryon asymmetry. On the other hand, values of $M_{N}$ up to $10^{10} \mathrm{GeV}$ at least can be accommodated if $\Gamma_{N} \simeq|B|$ and $\Im \mathrm{m}\left[A_{\nu}\right] \simeq 1 \mathrm{TeV}$.

There is yet another constraint on this scenario. Here $\tilde{N}$ particles are not produced at rest, so that their decay is delayed by a time dilatation factor $\sim m_{\phi} / M_{N}$. For given $\Gamma_{N}$ and $T_{\mathrm{R}}$ this would reduce the temperature at the time of $\tilde{N}$ decay, leading to a large reduction of $\left|\Delta_{F B}\right|$, see Eq.(23). Raising $T_{\mathrm{R}}$ is not an option, since then inverse $\tilde{N}$ decay reactions would become much more efficient, washing out any asymmetry. This scenario therefore requires the inflaton mass to be only a factor of a few larger than $M_{N}$.

Table 1 shows that the value of $M_{N}$ is less constrained in our scenario, and works for any inflaton mass $>M_{N}$. Here sneutrinos exist in the form of a condensate and there is no need to produce them after inflation. On the other hand, the constraints (33) and (30) require rather small values of $\bar{m}$ in our scenario, as remarked above.

\section{Additional thermal effects}

Note first that the existence of a thermal bath at $H>\Gamma_{\phi}$, with temperature $T>T_{\mathrm{R}}$, does not contradict the gravitino bound. The reason is that, for $\Gamma_{N}>\Gamma_{\phi}$, the Universe will become $\mathrm{RD}$ only later when reheating completes. This stage releases enough entropy to dilute any gravitinos produced at $T>T_{\mathrm{R}}$ [65].

The last rows in Table 1 have quite low values of $|B|$, well below $1 \mathrm{GeV}$. Such values may be natural in models of gauge-mediated supersymmetry breaking, where one might expect the gravitino mass to be near $|B|$. In this case the scaled relic density of gravitinos is given by $[66,67]$ :

$$
\Omega_{3 / 2} h^{2} \simeq 0.8\left(\frac{M_{3}}{1 \mathrm{TeV}}\right)^{2}\left(\frac{10 \mathrm{MeV}}{m_{3 / 2}}\right)\left(\frac{T_{\mathrm{R}}}{10^{6} \mathrm{GeV}}\right),
$$

where $h \simeq 0.7$ is the present scaled Hubble constant, and $M_{3}$ is the gluino mass. Then, for $M_{3} \sim 500 \mathrm{GeV}$, the dark matter limit leads to the constraint $T_{\mathrm{R}} \leq 10^{8} \mathrm{~m}_{3 / 2}$. We thus see that we need $m_{3 / 2} \gg|B|$ in the last two examples shown in Table 1, i.e. we have to require that the leading (in inverse powers of $M_{\mathrm{P}}$ ) operator that could give rise to a $B$-term is suppressed.

Note that the instantaneous thermal bath may well have a temperature $\gg M_{N}$ at early times, and can in principle produce $\tilde{N}$ and $\tilde{N}^{*}$ quanta with a number density $n_{\tilde{N}, t h} \simeq 0.2 T^{3}$. This may be larger than the number density of the zero-mode $\tilde{N}$ quanta in the condensate $n_{\tilde{N}, \text { con }} \simeq H^{2} N_{0}^{2} / M_{N}$ (which is typically much 
smaller than $T^{3}$ ). One might therefore wonder whether the former could yield a lepton asymmetry, according to $[36,37,12]$, dominating that generated by the latter. Eq. (36) shows that this will not be the case so long as $n_{\tilde{N}, t h} / n_{\tilde{N}, \text { con }}<M_{N} /\left|A_{\nu}\right|$ when $H \simeq|B|$ (note that at the time of sneutrino decay $n_{\tilde{N}, t h}<T^{3}$ ). Indeed, for all the examples in Table 1, the asymmetry generated by the condensate is dominant.

\section{Effects of additional RH (s)neutrinos}

So far we have considered only one of the sneutrinos. Indeed, one of the merits of this scenario is that it works well with just one generation of RH (s)neutrinos. Nevertheless, the other sneutrinos can also acquire a VEV during inflation and their post-inflationary dynamics may contribute to and/or affect the generated baryon asymmetry. Let us denote the three sneutrinos by $\tilde{N}_{1}, \tilde{N}_{2}, \tilde{N}_{3}$ with masses $M_{1}<M_{2}<M_{3}$ respectively. If $M_{i}>H_{I}$, $\tilde{N}_{i}$ will not develop a VEV thus being irrelevant for the production of the asymmetry. If $H_{I} \gtrsim 10^{13} \mathrm{GeV}$ as in simple models of inflation [21], these (s)neutrinos will then also be too heavy to wash out the asymmetry.

It is conceivable that all sneutrino fields whose mass $M_{i}<H_{I}$ have the same value $N_{0}$ (up to fluctuations of size $H_{I} \ll N_{0}$ ) after inflation ${ }^{\dagger \dagger}$; these field values will remain essentially constant until $H=M_{i}$, at which point the amplitude of $\tilde{N}_{i}$ begins to be redshifted $\propto 1 / t$. The number density of $\tilde{N}_{i}$ at $H \simeq M_{1}$ will therefore be $n_{i}=\left(M_{1}^{2} / M_{i}\right) N_{0}^{2}$. In this case $\tilde{N}_{1}$ would be the best candidate to yield the maximum lepton asymmetry. Then, however, $\tilde{N}_{2}$ and $\tilde{N}_{3}$ should not decay too fast such that the resulting thermal effects destroy the coherence of $\tilde{N}_{1}$. On the other hand, if the asymmetry is generated from $\tilde{N}_{3}$, then $\tilde{N}_{1}$ and $\tilde{N}_{2}$ should decay before dominating the Universe. Otherwise, such a late decay will dilute the asymmetry. An efficient leptogenesis from $\tilde{N}_{2}$ requires that $\tilde{N}_{3}$ decay not destroy the $\tilde{N}_{1}$ condensate and $\tilde{N}_{1}$ decay not dilute the generated asymmetry.

Finally, independently of whether lighter RH sneutrinos (if any) have a large VEV during inflation, they could dilute the asymmetry through scattering reactions, analogous to the discussion of "inverse decays" of the sneutrino that produced the asymmetry in the first place. This discussion shows that the most dangerous situation occurs when the temperature is near the mass of these lighter sneutrinos. If this mass is less than the reheat temperature, it follows that the effective mass $\bar{m}_{\text {light }}$ associated with these light sneutrinos should be below $\sim 0.1$

\footnotetext{
${ }^{\dagger \dagger}$ This, for example, naturally happens if the minimum of the sneutrino potential is set by a negative Hubble-induced soft mass $^{2}$ and $D$-terms under which the sneutrinos are nonsinglet.
}

$\mathrm{eV}$; not surprisingly, this is essentially the same bound one derives from analogous arguments in standard thermal leptogenesis [9]. This bound can be relaxed by an order of magnitude or so of the lighter neutrino masses lie above $T_{\mathrm{R}}$, thereby allowing to saturate the phenomenological bound (35), which of course applies to the effective neutrino masses associated with any of the $\mathrm{RH}$ neutrino fields.

We conclude that our scenario works most comfortably when the dynamics of the other sneutrinos does not affect the picture presented in previous sections. Then the generated baryon asymmetry will be given by the expression in (28), with $\tilde{N}$ being the sneutrino which results in the maximum asymmetry. The decay or exchange of other sneutrinos can suppress the produced asymmetry, thus constraining the scenario.

\section{E. Late decaying sneutrino}

So far we have assumed that the sneutrino decays before the inflaton does. If $\Gamma_{N}<\Gamma_{\phi}$ our scenario will not be altered qualitatively, so long as $\tilde{N}$ does not dominate the energy density of the Universe (see the next Subsection). Since the co-moving entropy density after inflaton decay is essentially constant in this case, the dilution factor (25) is the same as for $\Gamma_{N}>\Gamma_{\phi}$. The main difference is that the temperature decreases much faster in the radiation-dominated era: $T \propto t^{-1 / 2}$, as compared to $T \propto t^{-1 / 4}$ during the matter-dominated era after inflation. This means that there is a relatively shorter time window where $T \sim M_{N}$, which yields the maximal $\left|\Delta_{B F}\right|$. As a result, the effective $\left|\Delta_{B F}\right|$ will be slightly smaller than in scenarios with $\Gamma_{N}>\Gamma_{\phi}$. Moreover, for given temperature* the Hubble parameter in the matter-dominated epoch exceeds that in the radiationdominated era by a factor $\left(T / T_{\mathrm{R}}\right)^{2}$. Wash-out reactions are therefore more dangerous if $\Gamma_{N}<\Gamma_{\phi}$.

\section{F. $\tilde{N}$ as the inflaton or curvaton}

So far our estimates of the baryon asymmetry were based on the assumption that $\tilde{N}$ decays before dominating the Universe. In fact, when $|B| \simeq m_{0}=1 \mathrm{TeV}$, this will be necessary to avoid gravitino overproduction. However, $\tilde{N}$ domination is possible if the sneutrino is the inflaton, or the curvaton $[68,69]$. Then the maximum lepton asymmetry will be given by

$$
\frac{L_{D}}{s} \simeq \frac{3 T_{\mathrm{R}}}{4 M_{N}} \Delta_{B F},
$$

\footnotetext{
${ }^{*}$ Recall that in the matter-dominated era the hot plasma contributes only a small fraction to the total energy density.
} 
where $\Delta_{B F}$ is given by $(21)$ at $T=T_{\mathrm{R}}$. Here $T_{\mathrm{R}} \simeq 1.4\left(g_{*}^{-1 / 2} \Gamma_{N} M_{\mathrm{Pl}}\right)^{1 / 2}$ is the reheat temperature of the Universe after the decay of $\tilde{N}$. Note that for $T_{\mathrm{R}}<M_{N} / 30$, we simply have $\left|\Delta_{B F}\right| \approx\left(T_{\mathrm{R}} / M_{N}\right)^{2}$, see Eq. (23).

If $\tilde{N}$ is the inflaton $[60,70], M_{N}=10^{13} \mathrm{GeV}$ (to have density perturbations of the correct size) and, due to the gravitino bound $T_{\mathrm{R}} \leq 10^{9} \mathrm{GeV}, \Gamma_{N} \leq 10 \mathrm{GeV}$. Therefore obtaining the maximum asymmetry requires that $|B| \leq 10 \mathrm{GeV}$. Even then, the asymmetry will be too small by two orders of magnitude. A sufficient asymmetry can be generated with a higher $T_{\mathrm{R}}$ at the expense of gravitino overproduction. Then, however, late entropy production will be necessary in order to dilute the excess of gravitinos. Note that $\left(L_{D} / s\right) \propto T_{\mathrm{R}}^{3}$, see Eq. (40), while $\left(n_{3 / 2} / s\right) \propto T_{\mathrm{R}}$ [14]. Entropy release can therefore dilute gravitinos while yielding an acceptable asymmetry. For example, if $\Gamma_{N}=|B|=1 \mathrm{TeV}$ and $T_{\mathrm{R}}=10^{11}$ $\mathrm{GeV}$, successful leptogenesis will require a dilution factor of $10^{4}$.

If $\tilde{N}$ is the curvaton $[31,48,49], M_{N}$ and $T_{\mathrm{R}}$ can be much smaller. Then a sufficient asymmetry can be generated, provided that $10^{3} T_{\mathrm{R}} \geq M_{N}$, where $T_{\mathrm{R}}$ is the temperature of the Universe after $\tilde{N}$ decay. In this case the sneutrino dominance typically requires a tiny $h$ and a $N_{0}$ not much smaller than $M_{\mathrm{P}}$. This implies that $\Gamma_{N} \ll m_{0}$, and hence $|B| \ll m_{0}$ is required to have the maximum asymmetry in the condensate. A larger $|B|$ can be compensated for by appropriate increase in $T_{\mathrm{R}} / M_{N}$.

\section{SUMMARY AND CONCLUSIONS}

In this paper we have discussed a new variant of the leptogenesis mechanism for the creation of the baryon number of the Universe. Our starting point is the observation that the complex scalar field describing the superpartners of $S U(2) \times U(1)_{Y}$ singlet ("right-handed") neutrinos, introduced to explain the smallness of the observed neutrino masses through the see-saw mechanism, should acquire large expectation values during inflation, if their mass is less than the Hubble scale during inflation. Note that this argument applies to the real and imaginary parts of this field, i.e. one expects CP to be broken spontaneously in such a scenario. In addition, lepton number is violated in the scalar sector by the soft supersymmetry breaking $B$-term associated with the (supersymmetric) Majorana mass term for the heavy neutrinos. The third Sakharov condition is automatically satisfied, since the creation and decay of such a condensate is a non-equilibrium process.

We showed that such a scenario can indeed create the required baryon asymmetry if a number of conditions are satisfied. To begin with, the $B$ - term creates an oscillating lepton number in the condensate. This can be transferred efficiently to light (s)particles only if the period of this oscillation, given by $|B|$, is not too different from the decay width $\Gamma_{N}$ of the heavy sneutrino. Moreover, this transfer requires supersymmetry to be broken, the dominant contribution coming from thermal effects. This requires that the re-heat temperature $T_{\mathrm{R}}$ should not be too much below the mass $M_{N}$ of the heavy (s)neutrinos. Moreover, the condition that the sneutrino condensate should not be destroyed by thermal effects imposes an upper bound (33) on $T_{\mathrm{R}}$. On the other hand, the lower bound (30) on $T_{\mathrm{R}}$ can be derived if one requires that thermal effects should shut off possible non-perturbative $\tilde{N}$ decay mechanisms. As discussed in Sec. IV A, this may not be necessary, since other effects may prevent too early non-perturbative decays of the $\tilde{N}$ condensate. Recall also that non-perturbative effects might even increase the efficiency of our mechanism. However, in the absence of a complete quantitative understanding of these nonperturbative effects, we focused on scenarios which can be described purely perturbatively. In that case the condition of sufficient baryogenesis alone imposes both upper and lower bounds on $T_{\mathrm{R}}$, both of which scale $\propto M_{N}$ as discussed in Sec. V. This is to be contrasted with thermal leptogenesis, which only leads to a lower bound on $T_{\mathrm{R}}$ - which, however, is uncomfortably close to the upper bound on $T_{\mathrm{R}}$ from gravitino overproduction.

Note that $T_{\mathrm{R}}<M_{N}$ is required if $\tilde{N}$ is to decay before the inflaton does; we saw in Sec. VI E that in the opposite situation our mechanism will be somewhat less efficient. Our scenario therefore prefers $T_{\mathrm{R}} \lesssim 0.1 M_{N}$, whereas conventional thermal leptogenesis works best if $T_{\mathrm{R}} \gtrsim M_{N}$. Given the bound $T_{\mathrm{R}} \lesssim 10^{9}$ to avoid overproduction of gravitinos, our mechanism can accommodate significantly larger values of $M_{N}$, up to a few times $10^{10}$ $\mathrm{GeV}$; however, again in contrast to thermal leptogenesis, it can work also with much smaller $M_{N}$, so long as $|B|$ and the Yukawa coupling $h$ are reduced as well. On the other hand, if non-perturbative $\tilde{N}$ decays are shut off by thermal effects, our scenario requires the effective light neutrino mass associated with this particular heavy (s)neutrino to lie in a rather narrow range around $1 \mathrm{meV}$. The smallness of the Yukawa coupling also means that the heavy (s)neutrino superfield responsible for leptogenesis in this mechanism does not leave any imprint on the low-energy sparticle spectrum, and will therefore not contribute at any appreciable level to slepton-mediated lepton flavor violating processes. However, since our scenario works for a single heavy (s)neutrino (which may only couple to a single light neutrino), little can be said about the contributions of the other heavy neutrinos to the see-saw mass matrix of the light neutrinos. This should allow to construct models that incorporate our mechanism and yield a realistic mass matrix for the light neutrinos.

The main numerical results of our paper are Eq.(28), which adequately describes the final baryon asymmetry if the $\tilde{N}$ decay width perfectly matches the frequency with which the lepton number of the $\tilde{N}$ condensate oscillates, 
and Fig. 2, which shows how a mismatch between these two quantities reduces the baryon asymmetry. This last figure is qualitatively similar to the dependence of $n_{B}$ on the effective light neutrino mass in models of thermal leptogenesis.

The perhaps least appealing aspect of this mechanism is that the final result depends (quadratically) on the absolute value $N_{0}$ of the field at the end of inflation, and (linearly) on the sin of its phase. The latter is naturally expected to be $\mathcal{O}(1)$, while the former could easily be sufficiently large for our purposes. On the other hand, it might be meaningful that the model is sufficiently constrained that it does not allow to generate a baryon asymmetry orders of magnitude larger than the observed one. We therefore conclude that this mechanism should be regarded on a par with other mechanisms for leptogenesis that have been suggested in the literature.

\section{ACKNOWLEDGEMENTS}

The authors wish to thank S. Davidson, A. Mazumdar, G. Moore, Y. Nir and M. Peloso for useful discussions and comments. The work of R.A. is supported by the National Sciences and Engineering Research Council of Canada. The research of M.D. is supported by "Sonderforschungsbereich 375 für Astro-Teilchenphysik" der Deutschen Forschungsgemeinschaft.

[1] C. L. Bennett et al., astro-ph/0302207; D. N. Spergel et al., astro-ph/0302209.

[2] For a review, see: K. A. Olive, G. Steigman and T. P. Walker, Phys. Rept. 333, 389 (2000)

[3] A. D. Sakharov, JETP Lett. B 91, 24 (1967).

[4] V. Kuzmin, V. A. Rubakov, and M. E. Shaposhnikov, Phys. Lett. B 155, 36 (1985).

[5] S. Yu. Khlebnikov and M. E. Shaposhnikov, Nucl. Phys. B 308, 885 (1985).

[6] M. Fukugita and T. Yanagida, Phys. Lett. B 174, 45 (1986).

[7] M. Gell-Mann, P. Ramond and R. Slansky, in Supergravity, eds. P. van Nieuwenhuizen and D. Z. Freedman (North Holland 1979); T. Yanagida, Proceedings of Workshop on Unified Theory and Baryon number in the Universe, eds. O. Sawada and A. Sugamoto (KEK 1979); R. N. Mohapatra and G. Senjanovic, Phys. Rev. Lett. 44, 912 (1980).

[8] M. A. Luty, Phys. Rev. D 45, 455 (1992).

[9] M. Plümacher, Z. Phys. C 74, 549 (1997), and Nucl. Phys. B 530, 207 (1998); W. Buchmüller and M. Plümacher, Phys. Rept. 320, 329 (1999), and Int. J. Mod. Phys. A 15, 5047 (2000).

[10] W. Büchmuller, P. Di Bari and M. Plümacher, Phys.
Lett. B 547, 128 (2002), and Nucl. Phys. B 665, 445 (2003), and hep-ph/0401240.

[11] S. Davidson, J. High Energy Phys. 0303, 037 (2003).

[12] G. F. Giudice, A. Notari, M. Raidal, A. Riotto and A. Strumia, hep-ph/0310123.

[13] A. Pilaftsis, Int. J. Mod. Phys. A 14, 1811 (1999); A. Pilaftsis and T. E. J. Underwood, hep-ph/0309342.

[14] M. Yu. Khlopov and A. D. Linde, Phys. Lett. B 138, 265 (1984); J. Ellis, J. E. Kim and D. V. Nanopoulos, Phys. Lett. B 145, 181 (1984); J. Ellis, D. V. Nanopoulos, K. A. Olive and S.-J. Rey, Astropart. Phys. 4, 371 (1996); for a recent calculation, see: M. Boltz, A. Brandenburg and W. Büchmuller, Nucl. Phys. B 606, 518 (2001).

[15] For a review, see: S. Sarkar, Rep. Prog. Phys. 59, 1493 (1996).

[16] A. L. Maroto and A. Mazumdar, Phys. Rev. Lett 84, 1655 (2000); R. Kallosh, L. Kofman, A. D. Linde and A. Von Proeyen, Phys. Rev. D 61, 103503 (2000); G.F. Giudice, I. I. Tkachev and A. Riotto, J. High Energy Phys. 9908, 009 (1999), and J. High Energy Phys. 9911, 036 (1999).

[17] R. Allahverdi, M. Bastero-Gil and A. Mazumdar, Phys. Rev. D 64, 023516 (2001).

[18] H. P. Nilles, M. Peloso and L. Sorbo, Phys. Rev. Lett. 87, 051302 (2001), and J. High Energy Phys. 0104, 004 (2001).

[19] H. P. Nilles, K. A. Olive and M. Peloso, Phys. Lett. B 522, 304 (2001).

[20] R. Allahverdi, K. Enqvist and A. Mazumdar, Phys. Rev. D 65, 103519 (2002).

[21] For reviews on inflation, see: A. D. Linde, Particle Physics and Inflationary Cosmology, Harwood (1990); D. H. Lyth and A. Riotto, Phys. Rep. 314, 1 (1999).

[22] G. Lazarides and Q. Shafi, Phys. Lett. B 258, 305 (1991); K. Kumekawa, T. Moroi and T. Yanagida, Prog. Theor. Phys. 92, 437 (1994); T. Asaka, K. Hamaguchi, M. Kawasaki and T. Yanagida, Phys. Lett. B 464, 12 (1999), and Phys. Rev. D 61, 083512 (2000).

[23] G. F. Giudice, M. Peloso, A. Riotto and I. I. Tkachev, J. High Energy Phys. 9908, 014 (1999);

[24] J. Garcia-Bellido and E. Ruiz Morales, Phys. Lett. B 536, 193 (2002).

[25] L. Bento and Z. Berezhiani, Phys. Rev. Lett. 87, 231304 (2001).

[26] R. Allahverdi and A. Mazumdar, Phys. Rev. D 67, 023509 (2003).

[27] T. Dent, G. Lazarides and R. Ruiz de Austri, hep$\mathrm{ph} / 0312033$.

[28] B. A. Campbell, S. Davidson and K. A. Olive, Nucl. Phys. B 399, 111 (1993).

[29] L. Boubekeur, S. Davidson, M. Peloso and L. Sorbo, Phys. Rev. D 67, 043515 (2003).

[30] H. Murayama and T. Yanagida, Phys. Lett. B 322, 349 (1994).

[31] K. Hamaguchi, H. Murayama and T. Yanagida, Phys. Rev. D 65, 043512 (2002).

[32] Z. Berezhiani, A. Mazumdar and A. Pérez-Lorenzana, Phys. Lett. B 518, 282 (2001).

[33] I. Affleck and M. Dine, Nucl. Phys. B 249, 361 (1985).

[34] For a review, see: K. Enqvist and A. Mazumdar, Phys. 
Rept. 380, 99 (2003).

[35] R. Allahverdi, B. Dutta and A. Mazumdar, Phys. Rev. D 67, 123515 (2003).

[36] Y. Grossman, T. Kashti, Y. Nir and E. Roulet, Phys. Lett. Rev. 91, 251801 (2003).

[37] G. D'Ambrosio, G. F. Giudice and M. Raidal, Phys. Lett. B 575, 75 (2003).

[38] For a review on supersymmetry, see: H. P. Nilles, Phys. Rept. 110, 1 (1984).

[39] M. Dine, L. Randall and S. Thomas, Phys. Rev. Lett. 75, 398 (1995).

[40] R. Allahverdi, B. A. Campbell and K. A. Olive, Phys. Lett. B 341, 166 (1994).

[41] M. Dine, L. Randall and S. Thomas, Nucl. Phys. B 458, 291(1996).

[42] E. W. Kolb and M. S. Turner, The Early Universe, Addison-Wesley, 1990.

[43] M. Senami and K. Yamamoto, Phys. Rev. D 67, 095005 (2003).

[44] R. Allahverdi, M. Drees and A. Mazumdar, Phys. Rev. D 65, 065010 (2002).

[45] D. V. Nanopoulos and R. Rangarajan, Phys. Rev. D 64, 063511 (2001).

[46] A. Mazumdar and A. Pérez-Lorenzana, Phys. Rev. D 65, 107301 (2002), R. Allahverdi, K. Enqvist, A. Mazumdar and A. Pérez-Lorenzana, Nucl. Phys. B 618, 277 (2001).

[47] S. Davidson and S. Sarkar, J. High Energy Phys. 0011, 012 (2000); R. Allahverdi and M. Drees, Phys. Rev. D 66, 063513 (2002).

[48] T. Moroi and H. Murayama, Phys. Lett. B 553, 126 (2003).

[49] J. McDonald, Phys. Rev. D 68, 043505 (2003).

[50] A. Dolgov and A. D. Linde, Phys. Lett. B 116, 329 (1982); L. F. Abbott, E. Farhi and M. Wise, ibid. 117, 29 (1982).

[51] L. Kofman, A. D. Linde and A. A. Starobinsky, Phys. Rev. Lett. 73, 3195 (1994), and Phys. Rev. D 56, 3258 (1997).

[52] Y. Shtanov, J. Traschen and R. Brandenberger, Phys. Rev. D 51, 5438 (1995).

[53] M. Postma, and A. Mazumdar, JCAP 0401, 005 (2004).

[54] G. Felder and L. Kofman, Phys. Rev. D 63, 103503 (2001).

[55] R. Allahverdi and B. A. Campbell, Phys. Lett. B 395, 169 (1997).

[56] T. Prokopec and T. G. Roos, Phys. Rev. D 55, 3768 (1997).

[57] R. Allahverdi, B. A. Campbell and R. H. A. David Shaw, Phys. Lett. B 473, 246 (2000).

[58] E. W. Kolb, A. Notari and A. Riotto, Phys. Rev. D 68, 123505 (2003).

[59] R. Allahverdi, B. A. Campbell and J. Ellis, Nucl. Phys. B 579, 355 (2000); A. Anisimov and M. Dine, Nucl. Phys. B 619, 729 (2001).

[60] H. Murayama, H. Suzuki, T. Yanagida and J. Yokoyama, Phys. Rev. D 50, 2356 (1994).

[61] Some recent analyses of neutrino oscillations are: $M$. Maltoni, T. Schwetz, M. A. Tortola and J. W. F. Valle, Phys. Rev. D 68, 113010 (2003); A. Bandyopadhyay, S. Choubey, S. Goswami, S. T. Petcov and D. P. Roy, hep- ph/0309174; C. Giunti and M. Laveder, hep-ph/0310238.

[62] J. Hisano, T. Moroi, K. Tobe and M. Yamaguchi, Phys. Rev. D 53, 2442 (1996); J. Hisano, M. Nojiri, Y. Shimizu and M. Tanaka, Phys. Rev. D 60, 055008 (1999); J. A. Casas and A. Ibarra, Nucl. Phys. B 618, 171 (2001).

[63] J. Arafune, M. Koike and J. Sato, Phys. Rev. D 56, 3093 (1997), Erratum-ibid. D 60, 119905 (1999); K. Dick, M. Freund, M. Lindner and A. Romanino, Nucl. Phys. B 562, 29 (1999).

[64] T. Ibrahim and P. Nath, Phys. Rev. D 57, 748 (1998), Erratum-ibid. [D 58, 019901 (1998), D 60, 079903 (1999), D 60, 119901 (1999)]; M. Brhlik, G. J. Good and G. L. Kane, Phys. Rev. D 59, 115004 (1999).

[65] See the last reference in [16].

[66] T. Moroi, H. Murayama and M. Yamaguchi, Phys. Lett. B 303, 289 (1993).

[67] A. de Gouvêa, T. Moroi and H. Murayama, Phys. Rev. D 56, 1281 (1997).

[68] D. H. Lyth and D. Wands, Phys. Lett. B 524, 5 (2002); D. H. Lyth, C. Ungarelli and D. Wands, Phys. Rev. D 67, 023503 (2003).

[69] T. Moroi and T. Takahashi, Phys. Lett. B 522, 215 (2001), Erratum-ibid B 539, 303 (2002); Phys. Rev. D 66, 063501 (2002).

[70] J. Ellis, M. Raidal and T. Yanagida, Phys. Lett. B 581, 9 (2004). 